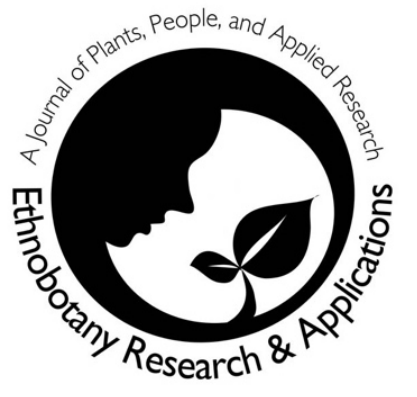

\section{Plants and fungi in the ethnomedicine of the medieval East - a review}

Karimjon Tayjanov, Olim Khojimatov, Yusufjon Gafforov, Trobjon Makhkamov, Rainer W. Bussmann and Nodirali Normakhamatov

\author{
Correspondence \\ Karimjon Tayjanov ${ }^{1}$, Olim Khojimatov ${ }^{1}$, Yusufjon Gafforov ${ }^{1,{ }^{*}}$, Trobjon Makhkamov ${ }^{2}$, Rainer W. Bussmann ${ }^{3^{*}}$ \\ and Nodirali Normakhamatov ${ }^{4}$ \\ ${ }^{1}$ Institute of Botany, Academy of Sciences of the Republic of Uzbekistan, 32 Durmon Yuli, Tashkent 100125, \\ Uzbekistan \\ ${ }^{2}$ Tashkent State Agrarian University, 2A Universitet, Tashkent region 100700, Uzbekistan \\ ${ }^{3}$ Department of Ethnobotany, Institute of Botany and School of Natural Sciences and Medicine Ilia State University, \\ Tbilisi 0105, Georgia \\ ${ }^{4}$ Tashkent Pharmaceutical Institute, 45 Aybek, Tashkent, 100015, Uzbekistan \\ *Corresponding Authors: Yusufjon Gafforov, yugafforov@yahoo.com, Rainer W. Bussmann, \\ rainer.bussmann@iliauni.edu.ge \\ Ethnobotany Research \& Applications 22:46 (2021)
}

\title{
Review
}

\begin{abstract}
Background. In the history of medicinal science of the medieval East, the merits of the great tabibs-physicians ArRazi, Avicenna (Ibn Sino), the scientist-encyclopedist Beruni, Yusufi and others are enormous. When treating patients, they used medicinal plants, as well as fungi, animal (ornithological) and mineral products. Such use of plants for medicinal purposes goes back centuries. In this review, we present a compilation of the most important plant species and fungi used in the traditional medicine of the medieval East.
\end{abstract}

Methods. We have obtained ethnomedicinal information by searching various resources, such as scientific and popular journal papers, monographs, books written in Uzbek, Russian and English, and our materials focused on the traditional use of medicinal plants and fungi. Scientific names, both of plants and fungi were checked for potential synonyms in the databases Index Fungorum and Plants of the World Online (POWO).

Results. Based on literary data on taxonomy, resource science, as well as folk and scientific medicine, we compiled a list of plant families used by healers and medicines of the Middle East. According to our results, 173 species of medicinal plants were used and described by Ar-Razi, 515 by Avicenna, 901 in Beruni, and 110 as well as a few fungal species in Yusufi. Among the medicinal plants used by Ar-Razi, Avicenna and the Beruni listed in Saidana are more than 240 species currently found in the flora of Uzbekistan.

Conclusion. On the face of the fact that the centuries-old experience of traditional medicine, generalized in the works of scientists from the Middle East, has found its confirmation at the present time. Most of the medicinal plants indicated by them are now included in the Pharmacopoeias of many countries, from which both independent medicinal products were obtained, and biologically active substances were isolated that serve in the improvement of the population. Given the growing interest in medicines of natural origin, work in this direction will continue.

Key words: Ethnobiology, Plants, Fungi, Folk Medicine, Scientific Medicine 


\section{Background}

People have known the healing and empowering properties of various herbs since ancient times, and they have used this knowledge in the treatment of various diseases. For example, in ancient Egypt, India, China, Central Asia and many other countries, physicians wrote many manuscripts on the healing properties of herbs. It is possible to find among them medicinal ingredients made from various herbs that have not lost their value and are used in practice (Iwu 1994; Hamed 2009; Ajmad et al. 2015; Malik et al. 2015; Raj et al. 2018; Souza et al. 2018; Živković et al. 2020).

Nowadays, everything is being forgotten faster and the memory of the folk traditional use of local plant species in different branches of a person's daily life, using them as amulets without saying already about the treatment of diseases, and their role in traditional folk rituals. That is why it is so important to be in time to collect and preserve, generalize and analyze this passing true folk knowledge (Tkachenko, Lebedeva 2008).

Eastern folk medicine or traditional medicine has held a special fascination for ethnographers, anthropologists, and ethnobiologists alike. Knowledge of folk medicine has been passed down via both written and oral pathways over centuries, and has been incorporated into Greek, Arab and other medical theories. While some of these medical traditions have survived the passage of time relatively intact, many others have changed or disappeared, while "new" remedies and uses have also emerged (Azaizeh et al. 2006).

Traditional medical knowledge is always in a state of flux. In many cases, local traditional knowledge regarding the environment, wild food and medicine sources, and human health are however in an alarming state of decline. This has prompted researchers to pursue studies to document, preserve and compare data concerning these unique local ethnomedical practices. In countries like Uzbekistan and in the Central Asian region, ethnobiological studies can enhance our understanding not only of traditional healthcare practices but also provide insight into human health and offer new solutions for food security. Specifically, ethnobiological data can provide a basis for understanding folk medical beliefs about sickness, health, and therapies that are useful to medical doctors charged with the care of populations that now mostly use allopathic treatments. Moreover, much research into the medicinal and nutritional value of plants and fungi those are presently underused in mainstream culture and may actually lead to the development of the foods, pharmaceuticals, and drugs of tomorrow (Ramalingum, Mahomoodally 2014; Howes et al. 2020).

\section{Materials and Methods}

Short information study site

In this article, Transoxiana is regarded as the region covering is the historical territories of Central Asia. The Transoxiana is located in Central Asia, between the Amudarya and Syrdarya rivers. The territory in the north and northeast borders with Kazakhstan, in the east and southeast - with Kyrgyzstan and Tajikistan, in the west - with Turkmenistan, in the south - with Afghanistan. Nowadays it covers the territories of five modern countries: Kazakhstan, Kyrgyzstan, Tajikistan, Turkmenistan, and Uzbekistan (Burghart and Sabonis-Helf 2018).

\section{The geographic location of Transoxiana}

This includes a desert located between the Amu Darya and Syr Darya river basins. The Transoxiana territories were occupied by a desert called Kyzyl-Kum. In the south and south-east regions are the Pamir-Alai and Tian-Shan mountains. The altitude ranges from $200 \mathrm{~m}$ in the desert to almost $6,000 \mathrm{~m}$ at the mountain peak. The riversides are densely populated by Kazakhs, Karakalpaks, Kyrgyz, Turkmens, and Uzbeks (Burghart and Sabonis-Helf 2018).

In the Middle Ages, Central Asia was inhabited mainly by Turks and Iranians. After the arrival of the Arabs in the region in 650-715 AD, Islam spread throughout the region and became an integral part of the culture of the people of the region. Medicine flourished in regions where Islam was widespread, and local people enriched it with their own medical traditions. Each of the plains, foothills, and mountainous regions of Central Asia has its own fauna, flora and fungi. It is from these gifts of nature that the healing culture of local people has grown, prescribing medicines for them Ibn Sina, Ar-Razi, and other scholars expected these medical traditions to reach the academic level (Lubin 1984).

\section{Central Asia Population}

Nowadays population of Central Asia is more than 75978668 people (https://www.worldometers.info/worldpopulation/central-asia-population/). Many nationalities and ethnic groups live here such as Uzbeks, Karakalpak's, Tajiks, Turkmens, Kazakhs, Kyrgyz, Uighurs, Dungan's; Western and Eastern Slavs: Russians, Ukrainians, Belarusians, 
Poles; numerous diasporas in five countries are Koreans, Iranians, Armenians, Georgians, Azerbaijanis, Tatars, Bashkir's, Germans, Jews, Lithuanians, Greeks, Turks, and many other nationalities (Lubin 1984)

\section{Data Collection}

We have obtained ethnomedicinal data from books written by Ar-Razi, Avicenna, Beruni, and Yusufi in the 10-11th and $16^{\text {th }}$ centuries, as well as various resources about their scientific works in scientific and popular journal papers, monographs, books written in Uzbek, Russian, English, and own materials, focused on the traditional use of medicinal plants and fungi. In addition, scientific names OF both plants and fungi were checked for potential synonyms in Index Fungorum (date accessed 10/04/2021) and Plants of the World Online (POWO) (date accessed: 09/07/2021) and a modern list of plants and fungi was compiled.

\section{Results and Discussion}

According to our results, to 173 species of medicinal plants were used and described by Ar-Razi, 515 by Avicenna, 901 in Beruni, and 110 in Yusufi. Medicinal plants and fungi mentioned in the works of Ar-Razi, Avicenna, al-Beruni and Yusufi are now found in the flora of many other countries around the world.

This information in this review was based on information from the books of Ar-Razi (Clinical records of Abu Bakr Razii 1994; Ar-Razi 1994; Taizhanov et al. 2003), Avicenna (Ibn Sina 1996), Beruni (Abu Raikhan Beruni 1973; Sobirov et al. 1993) and Yusufi (Khasani 1992; Toyzhanov, Kholmatov 1998), and other literary sources on folk and scientific medicine (Muravyova 1964; Wolf, Maleeva 1969), as well as our own materials. The names of the plants were given based on the Flora of Uzbekistan (Flora of Uzbekistan 1941-1962), Keys of Central Asian plants (Keys to plants of Central Asia 1968-1993) and Vascular Plants of the USSR (Cherepanov 1995). Many plant names may be etymologically related to the names of wild and domestic animals, birds, and insects. In the Uzbek language, there are plant names associated with the names of animals, such as "Otqulok - Horse" (Rumex) "Echkitol - Goat" (Salix) (Flora of Uzbekistan 1953), "Arslonquyruq - Lion" (Leonurus), "Burigul - Wolf" (Vinca) (Flora of Uzbekistan 1961), etc. In the fundamental key book "Flora of Uzbekistan" (Vol. 6.) there are more than 200 such Uzbek names of plants. The names of plants associated with the names of animals, birds and insects are also found in the works of Ar-Razi, Avicenna, Beruni and others (Sobirov et al. 1993; Khalmatov 1999; Taizhanov et al. 2003). The names of many medicinal plants in Arabic and Persian have been preserved in Uzbek language today (Khojimatov 2021).

\section{A short history of important scholars}

For thousands of years, the books of medieval medicinal scholars have been translated into many languages of the world. It is known that e.g., Avicenna's "al Qanun fit Tibb" in Arabic and "The Canon of Medicine" has been translated into Latin only more than 40 times.

Avicenna (Abu Ali Hussin bin Abdallah ibn Hasan ibn Ali ibn Sino) was born in 980 in the village of Afshana, present day Bukhara region in Uzbekistan. He is one of the outstanding encyclopedist scholars of the medieval East, who made a huge contribution to the history of world science and culture. His scientific heritage includes a variety of industries: philosophy, medicine, astronomy, mathematics, physics, poetry, music. In his autobiography, he writes "I didn't sleep all night, I didn't rest from morning till night, and I didn't do anything but mental work." (Ibn Sina 1996). The results of such work were reflected in his creative activity as a medical scientist. He lived and worked in many cities in Bukhara, Gurganj, Nishapur, Hamadan, Jurjan. As the ruler of Hamadan, he spent 9 years as a treating physician and vizier. In Hamadan, he began to write the "Book of Healing" (Kitab al-Shifa), then the first book with 5 volumes, "The Canon of Medical Science", which he finished all volumes in 1020. Numerous wanderings, continuous hard work exhausted the health of Avicenna. He died in 1037 in Hamadan. Avicenna's contribution to the development of medicine was especially significant, amounting to more than 55 books, of which 31 were written by Ibn Sina (Arzumetov, Yuldashev 1983). The "Canon" ("Kitab al-kanun fi-t-tib") - the main medical work of Avicenna is a genuine medical encyclopedia. For many centuries, this work of Avecinna served as the main medical guide of many countries, including in the East. The author himself divided the "Canon" into five books. The second book of the Canon is an encyclopedia of medicines. More than 800 medicinal agents of vegetable, mineral, and animal origin are described. It combines the experiments of medicine of ancient Rome, Greece, India, Iran and Central Asia (Karimov 1973). Of the 810 drugs listed in the second book of Canon, 515 are medicinal plants (and their agents), 125 products of animal origin, 85 minerals (Khalmatov 1958, 1999; Taizhanov, Taizhanova 2009). In addition to higher plants, it contains lichens (Leconora, Roccelia, Usnea) and fungi (Morchella, Fomitopsis, Tuber). Avicenna divides drugs into simple and complex ones. Simple consists of one plant (or resources), complex numbers from 4-6 to 10-15 items. Means are consisting of 60-80 plants and means of animal and mineral origin. In addition 
to drugs prepared by hand, he used the medicine of other authors, such as Barmacius (composition of 5 plants), Antil (7 plants), Andromachus (9 plants), Aristotle (25 plants), Mithridates (57 plant species).

Ar-Razi (Abu Bakr Muhammad ibn Zakariya Al Razi) was born in the city of Al Rayy in August 865 AD, and he died in Al Rayy on October 27, 925 at the age of 60 years. In his youth, he was fond of literature, music, philosophy, geography, astronomy, and other branches of science. However, he was engaged in the treatment of patients in older age. He lived only 60 years, but among more than 180 scientific papers 56 are devoted to treatment. In his book "Clinical Records of Abu Bakr Razi and His Disciple," as well as "Clinical Records" (both books were reprinted in Tashkent in 1974), the healing properties of 180 plant species belonging to 72 families are given in Uzbek (Clinical records of Abu Bakr Razii 1994; Ar-Razi 1994). In addition, he used more than 60 products of animal (including ornithological) and mineral origin (Clinical records of Abu Bakr Razii 1994; Ar-Razi 1994; Taizhanov et al. 2003).

Beruni (Abu Raihon Muhammad ibn Ahmad Al Beruniy) was born in Khorazm in Kiyat city in 973. He wrote more than 150 works devoted to almost all branches of knowledge of that time. Some works (Chronology, India, Geodesy, Canon Masuda) are published in translation into Russian, Uzbek and Western European languages and are available to a wide range of readers. Among Beruni's works, the last work "Kitab as Saidana fi-t-tibb" (abbreviated "Saidana") in terms of volume and content is a valuable source on the history of medicinal science in the medieval East (Abu Raikhan Beruni 1973). This work of Beruni differs from other works devoted to pharmacognosy, even from Avicenna's second book, The Canon of Medical Science, because it focuses not on the properties and effects of drugs, but on their definition, since in the medical literature of that time there were more foreign-language names of drugs that were not known to all doctors. He cited in work about 4,500 names of plants, animals, minerals, and other products obtained from them, in different languages of the people of the East of that time (Karimov 1973). Of the 1116 paragraphs given in the book, 901 plant species are devoted to medicinal herbs, 104 to animal products, 124 to minerals. However, the appendix contains information about 116 plants. Currently, of the plants reported by Beruni, 135 species are growing in the territory of Uzbekistan as wild, 89 species are as cultivated plants (Sobirov et al. 1993). Among them, 56 plant species are used in modern medicine and 114 in folk medicine (Taizhanov, Taizhanova 2009).

Yusufi (Yusufi ibn Muhammad ibn Yusuf al-tabib al Harawi) was born in the second half of the $16^{\text {th }}$ century in Khorasan. According to written sources, he learned to heal from his father. After arriving in Herat, he and his father worked in the "Medical House" ("Dorushshifo"), founded by the poet Alisher Navoi. He was one of the first physicians to investigate syphilis and healing of the disease with his medicines. He was one of the first doctors to diagnose syphilis and treat the disease with his own medications. After Babur (born in Andijan) conquered of India, he invited Yusufi to this country as a personal family physician. Among the works, he wrote on healing, "Jamiu-l-favoyid" ("Tibbi Yusufi") is a kind of lyrical essay - quadruple? ("Rubayat"), which gives the therapeutic properties of 108 wild and cultivated plants (Khasani 1992; Toyzhanov, Kholmatov 1998).

Based on literary data on taxonomy (Flora of Uzbekistan 1941-1962; Keys to plants of Central Asia 1968-1993; Cherepanov 1981), resource science (Wolf, Maleeva 1969), as well as folk and scientific medicine (Muravyova 1964; Sokolov, Zamotaev 1984), we compiled a list of plant families used by healers and medicines of the Middle East. In Table 1, taxa are given alphabetically according to the system of Angiosperm Phylogeny Group (APG IV 2016).

Among the medicinal plants used by Ar-Razi, Avicenna and the Beruni listed in Saidana are more than 240 (Ar-Razi 1994; Ibn Sina 1996; Beruni 1973) species currently found in the flora of Uzbekistan (Flora of Uzbekistan 1941-1962). These plants in Table 2 below are arranged in alphabetical order.

Thus, it was found that the medicinal plants used and cited in the works of Ar-Razi (149 genera with 173 species) (Clinical records of Abu Bakr Razii 1994; Ar-Razi 1994), Avicenna (365; 515 species) (Ibn Sina 1996), Beruni (505; 901 species) (Beruni 1973) and Yusufi (91; 110 species) (Khasani 1992) belong to 136 families (Fig. 1. and Table 1). Currently, these medicinal plants are found in the flora of more than 30 countries - this proposal has already been highlighted in many places, including in Uzbekistan (240 species), and are also used successfully in folk and scientific medicine. 
Table 1. List of plant families used by healers and medicines of the Middle East (Data from Sobirov et al. 1993, Khojimatov et al. 1995, Toyzhanov and Kholmatov 1998, Khalmatov 1999, Taizhanov and Taizhanova 2009, Taizhanov et al. 2003, Khojimatov 2021).

\begin{tabular}{|c|c|c|c|c|c|c|c|c|c|c|}
\hline \multirow[t]{3}{*}{ Family } & \multicolumn{8}{|c|}{ Scientist/Scholar } & \multicolumn{2}{|c|}{ Use in medicine } \\
\hline & \multicolumn{2}{|c|}{ Avicenna } & \multicolumn{2}{|l|}{ Ar-Razi } & \multicolumn{2}{|l|}{ Beruni } & \multicolumn{2}{|l|}{ Yusufi } & \multirow[t]{2}{*}{ Folk } & \multirow[t]{2}{*}{ Modern } \\
\hline & Genera & Species & Genera & Species & Genera & Species & Genera & Species & & \\
\hline Alismataceae & 1 & 2 & & & 1 & 1 & & & & \\
\hline Altingiaceae & 1 & 1 & & & 1 & 1 & & & & \\
\hline Amaranthaceae & 6 & 11 & 2 & 2 & 9 & 23 & 2 & 2 & 2 & \\
\hline Amaryllidaceae & 2 & 6 & 1 & 3 & 3 & 8 & 1 & 1 & 3 & \\
\hline Anacardiaceae & 3 & 5 & 3 & 4 & 4 & 7 & 3 & 3 & 5 & 4 \\
\hline Apiaceae & 29 & 40 & 13 & 13 & 36 & 63 & 5 & 8 & 18 & 8 \\
\hline Apocynaceae & 5 & 6 & & & 8 & 14 & & & & \\
\hline Araceae & 3 & 3 & 1 & 1 & 7 & 10 & 2 & 2 & 1 & 1 \\
\hline Araliaceae & 1 & 1 & 1 & 1 & 1 & 1 & & & & \\
\hline Arecaceae & 4 & 4 & & & 5 & 5 & 1 & 1 & & \\
\hline Aristolochiaceae & 2 & 3 & 2 & 2 & 2 & 3 & 2 & 3 & 2 & \\
\hline Asparagaceae & 7 & 7 & 2 & 2 & 7 & 10 & 1 & 1 & 2 & \\
\hline Asphodelaceae & 3 & 3 & 1 & 1 & 2 & 5 & 1 & 1 & 1 & 1 \\
\hline Aspleniaceae & 2 & 2 & & & 3 & 4 & 1 & 1 & 1 & 1 \\
\hline Asteraceae & 27 & 36 & 10 & 13 & 47 & 80 & 7 & 7 & 17 & 6 \\
\hline Berberidaceae & 2 & 3 & 1 & 1 & 2 & 4 & & & 1 & \\
\hline Betulaceae & 1 & 1 & 1 & 1 & 1 & 1 & 1 & 1 & 1 & \\
\hline Boraginaceae & 6 & 8 & 1 & 1 & 8 & 9 & 1 & 1 & 2 & \\
\hline Brassicaceae & 11 & 19 & 2 & 5 & 18 & 33 & 2 & 3 & 5 & 2 \\
\hline Burseraceae & 2 & 5 & 2 & 3 & 3 & 11 & 1 & 4 & 5 & 2 \\
\hline Buxaceae & & & & & 1 & 2 & & & & \\
\hline Campanulaceae & & & & & 1 & 1 & & & & \\
\hline Cannabaceae & 1 & 1 & & & 2 & 3 & & & 1 & \\
\hline Capparaceae & 1 & 1 & 1 & 1 & 3 & 3 & & & 1 & \\
\hline Caprifoliaceae & 2 & 3 & 1 & 1 & 2 & 5 & 1 & 1 & 1 & 1 \\
\hline Caryophyllaceae & 4 & 3 & 1 & 1 & 8 & 11 & & & 1 & \\
\hline Cistaceae & 1 & 2 & 1 & 1 & 1 & 3 & & & 1 & \\
\hline Combretaceae & 1 & 2 & 1 & 1 & 1 & 4 & 1 & 2 & 2 & 1 \\
\hline Convolvulaceae & 3 & 5 & 3 & 3 & 3 & 9 & 3 & 3 & 3 & 1 \\
\hline Cornaceae & 1 & 1 & & & 1 & 2 & & & & \\
\hline Costaceae & 1 & 1 & & & 1 & 1 & & & & \\
\hline Crassulaceae & 1 & 1 & & & 2 & 2 & & & & \\
\hline Cucurbitaceae & 5 & 7 & 4 & 5 & 8 & 17 & 1 & 1 & 5 & 1 \\
\hline Cupressaceae & 3 & 5 & 1 & 1 & 3 & 5 & & & 1 & 1 \\
\hline Cynomoriaceae & 1 & 1 & 1 & 1 & 1 & 1 & & & 1 & \\
\hline Cyperaceae & 1 & 4 & 1 & 2 & 1 & 4 & 1 & 2 & 2 & \\
\hline Dioscoreaceae & 1 & 1 & & & 1 & 1 & & & & \\
\hline Dipterocarpaceae & & & & & 1 & 1 & & & & \\
\hline Droseraceae & & & & & 1 & 1 & & & & \\
\hline Ebenaceae & 1 & 1 & & & 1 & 1 & & & & \\
\hline Elaeagnaceae & 1 & 1 & & & 1 & 1 & & & & \\
\hline Ephedraceae & & & & & 1 & $?$ & & & & \\
\hline Equisetaceae & 1 & 1 & & & 1 & 1 & & & & \\
\hline Ericaceae & & & & & 1 & 1 & & & & \\
\hline Erythroxylaceae & & & & & 1 & 1 & & & & \\
\hline Euphorbiaceae & 6 & 9 & 2 & 2 & 9 & 19 & 3 & 3 & 5 & 2 \\
\hline Fabaceae & 32 & 44 & 14 & 17 & 41 & 84 & 6 & 7 & 17 & 7 \\
\hline Fagaceae & 2 & 3 & 1 & 1 & 4 & 8 & & & 1 & \\
\hline Gentianaceae & 2 & 2 & 1 & 1 & 3 & 4 & 2 & 2 & 2 & 1 \\
\hline Geraniaceae & 1 & 1 & & & 1 & 1 & 1 & 1 & 1 & 1 \\
\hline Haloragaceae & & & & & 1 & 1 & & & & \\
\hline Hamamelidaceae & & & 1 & 1 & 1 & 1 & & & 1 & 1 \\
\hline Hypericaceae & 3 & 3 & 1 & 1 & 3 & 5 & & & 1 & \\
\hline Icacinaceae & & & & & 1 & 1 & & & & \\
\hline Iridaceae & 4 & 5 & 2 & 2 & 3 & 4 & 1 & 1 & 2 & 1 \\
\hline Juglandaceae & 1 & 1 & 1 & 1 & 1 & 1 & 1 & 1 & 1 & 1 \\
\hline Juncaceae & & & & & 1 & 4 & & & & \\
\hline
\end{tabular}




\begin{tabular}{|c|c|c|c|c|c|c|c|c|c|c|}
\hline \multirow[t]{3}{*}{ Family } & \multicolumn{8}{|c|}{ Scientist/Scholar } & \multicolumn{2}{|c|}{ Use in medicine } \\
\hline & \multicolumn{2}{|c|}{ Avicenna } & \multicolumn{2}{|l|}{ Ar-Razi } & \multicolumn{2}{|l|}{ Beruni } & \multicolumn{2}{|l|}{ Yusufi } & \multirow[t]{2}{*}{ Folk } & \multirow[t]{2}{*}{ Moderr } \\
\hline & Genera & Species & Genera & Species & Genera & Species & Genera & Species & & \\
\hline Lamiaceae & 14 & 31 & 7 & 9 & 19 & 43 & 3 & 3 & 9 & 6 \\
\hline Lauraceae & 2 & 5 & 2 & 4 & 4 & 12 & 2 & 3 & 4 & 3 \\
\hline Lecanoraceae & 1 & 3 & & & 1 & 2 & & & & \\
\hline Liliaceae & 4 & 5 & & & 4 & 7 & 1 & 1 & 3 & \\
\hline Linaceae & 1 & 1 & 1 & 1 & 1 & 1 & & & 1 & 1 \\
\hline Loganiaceae & & & 1 & 1 & 1 & 1 & & & 1 & 1 \\
\hline Loranthaceae & 1 & 1 & & & 1 & 1 & & & & \\
\hline Lythraceae & 3 & 3 & 1 & 1 & 4 & 5 & $?$ & 1 & 1 & \\
\hline Malpighiaceae & & & & & 1 & 1 & & & & \\
\hline Malvaceae & 7 & 7 & 4 & 4 & 7 & 10 & & & 4 & 2 \\
\hline Marsileaceae & & & & & 1 & 1 & & & & \\
\hline Melastomataceae & & & & & 2 & 3 & & & & \\
\hline Meliaceae & 1 & 1 & & & 3 & 3 & & & & \\
\hline Menispermaceae & 1 & 1 & & & 2 & 2 & & & & \\
\hline Montiniaceae & & & & & 1 & 1 & & & & \\
\hline Moraceae & 2 & 6 & 1 & 1 & 3 & 12 & & & 1 & 1 \\
\hline Morchellaceae & 1 & 1 & & & 1 & 1 & & & & \\
\hline Moringaceae & 1 & 2 & 1 & 1 & 1 & 2 & 1 & 2 & 1 & \\
\hline Musaceae & 1 & 1 & & & 1 & 2 & 1 & 1 & & \\
\hline Myristicaceae & 1 & 1 & & & 1 & 2 & 1 & 1 & 1 & 1 \\
\hline Myrtaceae & 3 & 3 & 2 & 2 & 3 & 3 & 1 & 1 & 2 & 2 \\
\hline Neuradaceae & & & & & 1 & 1 & & & & \\
\hline Nitrariaceae & 1 & 1 & & & 1 & 1 & & & & \\
\hline Nymphaeaceae & 1 & 2 & 1 & 1 & 4 & 7 & & & 1 & \\
\hline Oleaceae & 3 & 4 & 2 & 2 & 3 & 8 & $?$ & 1 & 3 & 2 \\
\hline Onagraceae & & & & & 1 & 1 & & & & \\
\hline Orchidaceae & 1 & 4 & & & 1 & 4 & 1 & 1 & 1 & 1 \\
\hline Oxalidaceae & & & & & 1 & 1 & & & & \\
\hline Paeoniaceae & 1 & 1 & & & 1 & 1 & & & & \\
\hline Pandanaceae & & & & & 1 & 2 & & & & \\
\hline Papaveraceae & 4 & 3 & 4 & 4 & 5 & 7 & 2 & 2 & 6 & 4 \\
\hline Pedaliaceae & 1 & 1 & 1 & 1 & 1 & 2 & 1 & 1 & 1 & 1 \\
\hline Penaeaceae & & & 1 & 1 & 1 & 1 & & & 1 & 1 \\
\hline Pinaceae & 4 & 7 & & & 3 & 6 & & & 1 & \\
\hline Piperaceae & 1 & 4 & 1 & 4 & 1 & 4 & 1 & 3 & 4 & 2 \\
\hline Plantaginaceae & 1 & 2 & 1 & 1 & 1 & 2 & & & 1 & 1 \\
\hline Platanaceae & 1 & 1 & & & 1 & 1 & & & & \\
\hline Plumbaginaceae & 2 & 2 & & & 1 & 2 & & & & \\
\hline Poaceae & 16 & 18 & 7 & 7 & 23 & 37 & 1 & 1 & 7 & 1 \\
\hline Polygonaceae & 3 & 7 & 2 & 2 & 6 & 13 & & & 1 & \\
\hline Polypodiaceae & 4 & 4 & 2 & 2 & 3 & 5 & 1 & 1 & 2 & 1 \\
\hline Polyporaceae & 1 & 1 & & & 1 & 1 & 1 & 1 & 1 & \\
\hline Portulacaceae & 1 & 2 & 1 & 1 & 1 & 2 & & & 1 & \\
\hline Potamogetonaceae & 1 & 1 & & & 1 & 1 & & & & \\
\hline Primulaceae & 5 & 4 & & & 5 & 5 & 1 & 1 & 1 & \\
\hline Pteridaceae & 1 & 1 & & & 2 & 2 & 1 & 1 & 1 & 1 \\
\hline Ranunculaceae & 6 & 8 & & & 7 & 14 & 2 & 2 & 2 & 1 \\
\hline Resedaceae & 1 & 1 & & & 1 & 1 & & & & \\
\hline Rhamnaceae & 2 & 3 & 1 & 1 & 2 & 5 & 1 & 1 & 2 & 2 \\
\hline Rhizophoraceae & & & & & 1 & 1 & & & & \\
\hline Roccelaceae & 1 & 1 & & & 1 & 1 & & & & \\
\hline Rosaceae & 12 & 20 & 6 & 6 & 11 & 30 & & & 6 & 2 \\
\hline Rubiaceae & 2 & 2 & 1 & 1 & 3 & 4 & & & 1 & 1 \\
\hline Rutaceae & 6 & 6 & 2 & 3 & 7 & 11 & 2 & 3 & 4 & 3 \\
\hline Salicaceae & 3 & 8 & & & 3 & 10 & 1 & 1 & 1 & \\
\hline Salvadoraceae & & & & & 1 & 1 & & & & \\
\hline Santalaceae & 1 & 1 & 1 & 1 & 3 & 3 & 1 & 1 & 1 & \\
\hline Sapindaceae & 1 & 1 & & & 1 & 1 & & & & \\
\hline Sapotaceae & 1 & 1 & & & 1 & 1 & & & & \\
\hline Scrophulariaceae & 3 & 5 & & & 3 & 5 & 1 & 1 & 1 & \\
\hline Smilacaceae & & & & & 1 & 1 & & & & \\
\hline
\end{tabular}




\begin{tabular}{|c|c|c|c|c|c|c|c|c|c|c|}
\hline \multirow[t]{3}{*}{ Family } & \multicolumn{8}{|c|}{ Scientist/Scholar } & \multicolumn{2}{|c|}{ Use in medicine } \\
\hline & \multicolumn{2}{|c|}{ Avicenna } & \multicolumn{2}{|l|}{ Ar-Razi } & \multicolumn{2}{|l|}{ Beruni } & \multicolumn{2}{|l|}{ Yusufi } & \multirow[t]{2}{*}{ Folk } & \multirow[t]{2}{*}{ Modern } \\
\hline & Genera & Species & Genera & Species & Genera & Species & Genera & Species & & \\
\hline Solanaceae & 6 & 9 & 3 & 3 & 8 & 19 & 2 & 2 & 4 & 1 \\
\hline Styracaceae & 1 & 1 & & & 1 & 1 & & & & \\
\hline Symplocaceae & 1 & 1 & & & 1 & 1 & & & & \\
\hline Tamaricaceae & 1 & 1 & 1 & 1 & 1 & 3 & 1 & 1 & 1 & \\
\hline Taxaceae & 1 & 1 & & & 1 & 1 & 1 & 1 & 1 & \\
\hline Theaceae & & & & & 2 & 2 & & & & \\
\hline Thymelaeaceae & 4 & 5 & 2 & 2 & 4 & 6 & 1 & 1 & 2 & \\
\hline Tuberaceae & 1 & 1 & & & 1 & 4 & & & & \\
\hline Typhaceae & & & & & 1 & 1 & & & & \\
\hline Ulmaceae & 1 & 2 & & & 2 & 2 & & & & \\
\hline Urticaceae & 2 & 4 & 1 & 1 & 2 & 4 & & & 1 & 1 \\
\hline Usneaceae & 1 & 1 & & & 1 & 2 & 1 & 1 & 1 & 1 \\
\hline Verbenaceae & 2 & 2 & & & 4 & 4 & 1 & 1 & 1 & \\
\hline Viburnaceae & & & & & 1 & 2 & & & & \\
\hline Violaceae & 1 & 1 & 1 & 1 & 1 & 1 & & & 1 & \\
\hline Vitaceae & 1 & 2 & 1 & 1 & 3 & 6 & 1 & 1 & 1 & 1 \\
\hline Zingiberaceae & 5 & 8 & 4 & 5 & 5 & 10 & 3 & 4 & 5 & 1 \\
\hline Zygophyllaceae & 2 & 2 & 1 & 1 & 3 & 4 & & & 1 & 1 \\
\hline Total & 365 & 515 & 149 & 173 & 505 & 901 & 91 & 110 & 214 & 91 \\
\hline
\end{tabular}

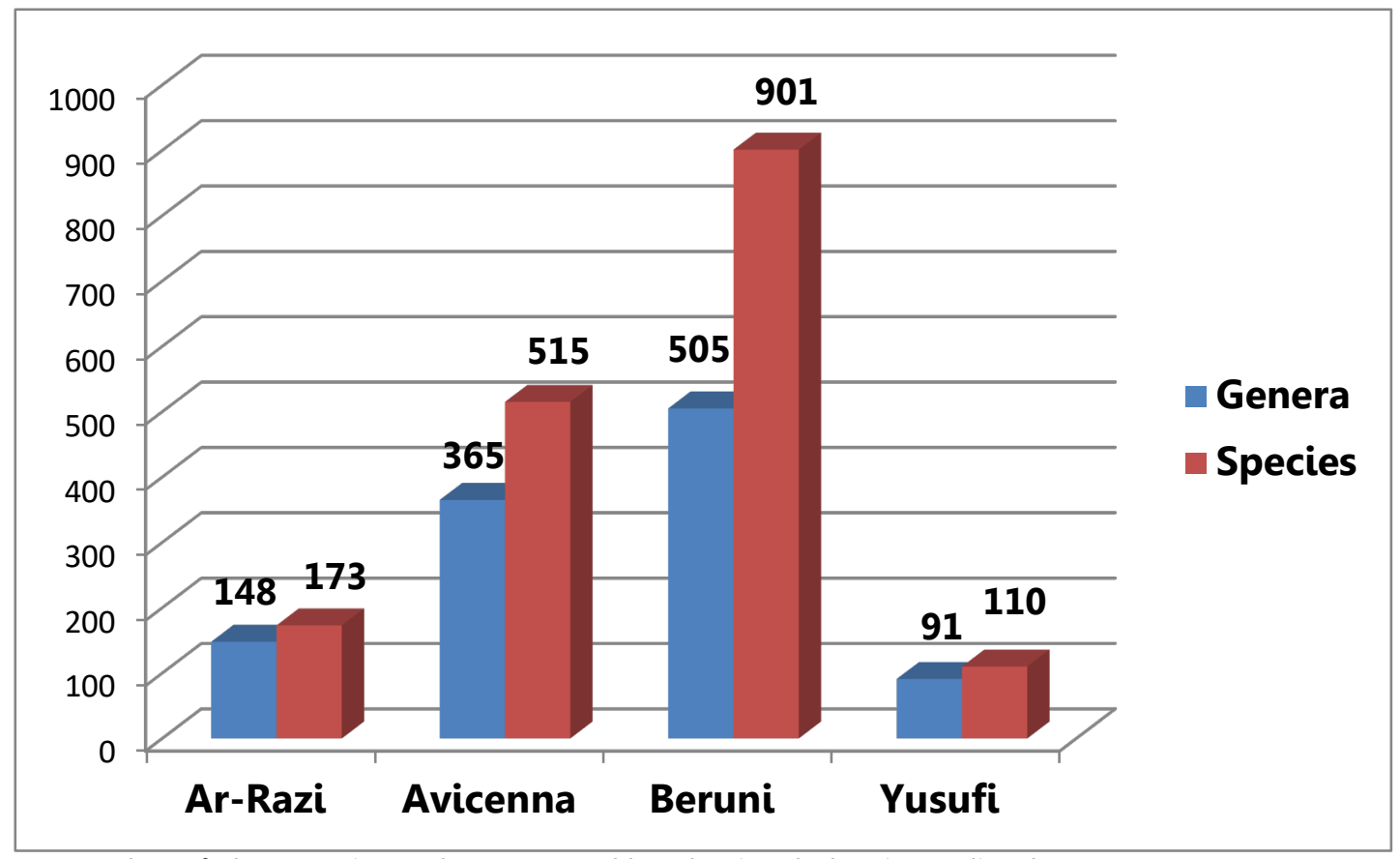

Figure 1. Number of plant species and genera used by Islamic scholars in medieval East.

Table 2. List of plant species used in traditional Islamic medicine. (Data from Sobirov et al. 1993, Khojimatov et al. 1995, Toyzhanov and Kholmatov 1998, Khalmatov 1999, Taizhanov and Taizhanova 2009, Taizhanov et al. 2003, Khojimatov 2021).

\begin{tabular}{|c|c|c|c|c|c|c|}
\hline \multirow[t]{2}{*}{ Plant species } & \multirow[t]{2}{*}{ Family } & \multirow[t]{2}{*}{ Ar-Razi } & \multirow[t]{2}{*}{ Avicenna } & \multirow[t]{2}{*}{ Beruni } & \multicolumn{2}{|c|}{ Use in medicine } \\
\hline & & & & & Folk & Modern \\
\hline Abutilon theophrasti Medik. & Malvaceae & & 1 & & 1 & \\
\hline Achillea millefolium $\mathrm{L}$. & Asteraceae & & & 1 & 1 & 1 \\
\hline Achillea tenuifolia Lam. & Asteraceae & & 1 & 1 & 1 & 1 \\
\hline Acorus calamus L. & Araceae & 1 & 1 & 1 & 1 & 1 \\
\hline Adianthum capillus-veneris $\mathrm{L}$. & Pteridaceae & 1 & 1 & 1 & 1 & 1 \\
\hline Aeluropus lagopoides (L.) Thwaites & Poaceae & & & 1 & & \\
\hline Agrostemma githago L. & Caryophyllaceae & & & 1 & 1 & \\
\hline Alhagi maurorum Medik. & Fabaceae & 1 & 1 & 1 & 1 & \\
\hline Alisma plantago-aquatica L. & Alismataceae & & 1 & 1 & 1 & \\
\hline
\end{tabular}




\begin{tabular}{|c|c|c|c|c|c|c|}
\hline \multirow[t]{2}{*}{ Plant species } & \multirow[t]{2}{*}{ Family } & \multirow[t]{2}{*}{ Ar-Razi } & \multirow[t]{2}{*}{ Avicenna } & \multirow[t]{2}{*}{ Beruni } & \multicolumn{2}{|c|}{ Use in medicine } \\
\hline & & & & & Folk & Modern \\
\hline Alkekengi officinarum Moench & Solanaceae & 1 & 1 & 1 & 1 & \\
\hline Allium ampeloprasum $\mathrm{L}$. & Amaryllidaceae & & 1 & 1 & 1 & \\
\hline Allium cepa L. & Amaryllidaceae & 1 & 1 & 1 & 1 & 1 \\
\hline Allium sativum $\mathrm{L}$. & Amaryllidaceae & 1 & 1 & 1 & 1 & 1 \\
\hline Aloe vera (L.) Burm. $\mathrm{f}$. & Xanthorrhoeaceae & 1 & 1 & 1 & 1 & 1 \\
\hline Althaea officinalis $\mathrm{L}$. & Malvaceae & 1 & 1 & 1 & 1 & \\
\hline Amaranthus blitum $\mathrm{L}$. & Amaranthaceae & & 1 & 1 & 1 & \\
\hline Amaranthus cruentus $\mathrm{L}$. & Amaranthaceae & & & 1 & 1 & \\
\hline Amaranthus tricolor $\mathrm{L}$. & Amaranthaceae & & 1 & 1 & 1 & \\
\hline Anchusa azurea Mill. & Boraginaceae & & 1 & 1 & 1 & \\
\hline Apium graveolens $\mathrm{L}$. & Apiaceae & 1 & 1 & 1 & 1 & 1 \\
\hline Artemisia absinthium L. & Asteraceae & 1 & 1 & 1 & 1 & 1 \\
\hline Artemisia dracunculus L. & Asteraceae & 1 & 1 & 1 & 1 & \\
\hline Artemisia vulgaris $\mathrm{L}$. & Asteraceae & 1 & 1 & 1 & 1 & \\
\hline Arundo donax $\mathrm{L}$. & Poaceae & & & 1 & & \\
\hline Asparagus officinalis $\mathrm{L}$. & Asparagaceae & 1 & 1 & 1 & 1 & 1 \\
\hline Asplenium ceterach $\mathrm{L}$. & Aspleniaceae & & 1 & 1 & 1 & \\
\hline Asplenium incisum Thunb. & Aspleniaceae & & 1 & 1 & & \\
\hline Avena sativa $\mathrm{L}$. & Poaceae & & 1 & 1 & 1 & 1 \\
\hline Berberis asiatica Roxb. ex DC. & Berberidaceae & 1 & 1 & 1 & 1 & 1 \\
\hline Berberis vulgaris $\mathrm{L}$. & Berberidaceae & & 1 & 1 & 1 & 1 \\
\hline Beta vulgaris $\mathrm{L}$. & Amaranthaceae & 1 & 1 & 1 & 1 & \\
\hline Brassica juncea (L.) Czern. & Brassicaceae & & 1 & & 1 & 1 \\
\hline Brassica nigra (L.) W.D.J. Koch & Brassicaceae & 1 & 1 & 1 & 1 & 1 \\
\hline Brassica oleracea L. & Brassicaceae & 1 & 1 & 1 & 1 & \\
\hline Brassica rapa L. & Brassicaceae & 1 & 1 & 1 & 1 & \\
\hline Bryonia alba L. & Cucurbitaceae & & 1 & 1 & 1 & 1 \\
\hline Bryonia dioca Bojer & Cucurbitaceae & & 1 & 1 & 1 & \\
\hline Buxus sempervirens $\mathrm{L}$. & Buxaceae & & & 1 & & \\
\hline Calendula officinalis $\mathrm{L}$. & Asteraceae & & 1 & 1 & 1 & 1 \\
\hline Calystegia sepium (L.) R. Br. & Convolvulaceae & & & 1 & 1 & \\
\hline Cannabis sativa $\mathrm{L}$. & Cannabaceae & & 1 & 1 & 1 & \\
\hline Capparis spinosa L. & Capparaceae & 1 & 1 & 1 & 1 & 1 \\
\hline Carthamus tinctorius $\mathrm{L}$. & Asteraceae & 1 & 1 & 1 & 1 & 1 \\
\hline Carum carvi $\mathrm{L}$. & Apiaceae & 1 & 1 & 1 & 1 & 1 \\
\hline Castanea sativa Mill. & Fabaceae & & & 1 & & \\
\hline Cedrus deodara (Roxb. ex D. Don) G. Don & Pinaceae & & & 1 & & \\
\hline Celtis australis $\mathrm{L}$. & Cannabaceae & & & 1 & & \\
\hline Cercis siliquastrum $\mathrm{L}$. & Fabaceae & & & 1 & & \\
\hline Chelidonium majus $\mathrm{L}$. & Papaveraceae & & 1 & 1 & 1 & 1 \\
\hline $\begin{array}{l}\text { Chenopodiastrum murale (L.) S. Fuentes, } \\
\text { Uotila \& Borsch }\end{array}$ & Amaranthaceae & & & 1 & & \\
\hline Chenopodium album $\mathrm{L}$. & Amaranthaceae & & & 1 & 1 & \\
\hline Chondrilla juncea L. & Asteraceae & & & 1 & 1 & \\
\hline Chrysojasminum fruticans (L.) Banfi & Oleaceae & & & 1 & & \\
\hline Cicer arietinum $\mathrm{L}$. & Fabaceae & 1 & 1 & 1 & 1 & \\
\hline Cichorium intybus $\mathrm{L}$. & Asteraceae & & 1 & 1 & 1 & 1 \\
\hline Citrullus colocynthis (L.) Schrad. & Cucurbitaceae & 1 & 1 & 1 & 1 & 1 \\
\hline Citrus $\times$ limon (L.) Osbeck & Rutaceae & 1 & & 1 & 1 & \\
\hline Conium maculatum $\mathrm{L}$. & Apiaceae & & 1 & 1 & 1 & 1 \\
\hline Convolvulus arvensis L. & Convolvulaceae & & 1 & 1 & 1 & \\
\hline Corchorus olitorius L. & Tiliaceae & & 1 & 1 & & 1 \\
\hline Coriandrum sativum $\mathrm{L}$. & Apiaceae & 1 & 1 & 1 & 1 & 1 \\
\hline Cormus domestica (L.) Spach & Rosaceae & & 1 & 1 & & \\
\hline Cornus mas L. & Cornaceae & & 1 & 1 & & \\
\hline Corylus avellana $\mathrm{L}$. & Betulaceae & 1 & 1 & 1 & 1 & \\
\hline Crataegus pontica K. Koch & Rosaceae & & 1 & 1 & 1 & \\
\hline Cucumis maxima Duchesne & Cucurbitaceae & 1 & 1 & 1 & 1 & 1 \\
\hline Cucumis melo L. & Cucurbitaceae & & 1 & & 1 & \\
\hline Cucumis sativus $\mathrm{L}$. & Cucurbitaceae & 1 & & 1 & 1 & \\
\hline Cuminum cyminum $\mathrm{L}$. & Apiaceae & 1 & 1 & 1 & 1 & \\
\hline Cupressus sempervirens $\mathrm{L}$. & Cupressaceae & & & 1 & & \\
\hline
\end{tabular}




\begin{tabular}{|c|c|c|c|c|c|c|}
\hline \multirow[t]{2}{*}{ Plant species } & \multirow[t]{2}{*}{ Family } & \multirow[t]{2}{*}{ Ar-Razi } & \multirow[t]{2}{*}{ Avicenna } & \multirow[t]{2}{*}{ Beruni } & \multicolumn{2}{|c|}{ Use in medicine } \\
\hline & & & & & Folk & Modern \\
\hline Cuscuta epithymum Siev. ex Ledeb. & Convolvulaceae & 1 & 1 & 1 & 1 & \\
\hline Cydonia oblonga Mill. & Rosaceae & 1 & 1 & 1 & 1 & \\
\hline Cynodon dactylon (L.) Pers. & Poaceae & & 1 & 1 & 1 & \\
\hline Cynomorium coccineum $\mathrm{L}$. & Cynomoriaceae & 1 & 1 & 1 & 1 & \\
\hline Cyperus maculatus Boeckeler & Cyperaceae & 1 & 1 & 1 & 1 & \\
\hline Cyperus rotundus $\mathrm{L}$. & Cyperaceae & 1 & 1 & 1 & 1 & \\
\hline Datura stramonium $\mathrm{L}$. & Solanaceae & & 1 & 1 & 1 & 1 \\
\hline Daucus carota L. & Apiaceae & 1 & 1 & 1 & 1 & 1 \\
\hline Delphinium semibarbatum Bien. ex Boiss. & Ranunculaceae & & & 1 & 1 & 1 \\
\hline Dictamnus albus $\mathrm{L}$. & Rutaceae & & 1 & 1 & & \\
\hline Dryopteris filix-mas (L.) Schott & Polypodiaceae & & 1 & 1 & & \\
\hline Ecballium elaterium (L.) A. Rich. & Cucurbitaceae & & 1 & 1 & 1 & \\
\hline Echinophora sibthorpiana Guss. & Apiaceae & & 1 & 1 & 1 & \\
\hline Elaeagnus angustifolia L. & Elaeagnaceae & & 1 & 1 & 1 & 1 \\
\hline Elymus repens (L.) Gould & Poaceae & & 1 & 1 & 1 & \\
\hline Equisetum arvense $\mathrm{L}$. & Equisetaceae & & 1 & 1 & 1 & 1 \\
\hline Eremurus olgae Regel & Asphodelaceae & & & 1 & 1 & \\
\hline $\begin{array}{l}\text { Eremurus stenophyllus subsp. aurantiacus } \\
\text { (Baker) Wendelbo }\end{array}$ & Asphodelaceae & & & 1 & 1 & \\
\hline Eruca vesicaria (L.) Cav. & Brassicaceae & & 1 & 1 & 1 & \\
\hline Eupatorium cannabinum $\mathrm{L}$. & Asteraceae & & 1 & 1 & 1 & \\
\hline Fagopyrum esculentum Moench & Polygonaceae & & & 1 & 1 & 1 \\
\hline Ferula assa-foetida $\mathrm{L}$. & Apiaceae & & 1 & 1 & 1 & 1 \\
\hline Ferula moschata (H.Reinsch) Koso-Pol. & Apiaceae & & 1 & 1 & 1 & \\
\hline Ficus carica $\mathrm{L}$. & Moraceae & 1 & 1 & 1 & 1 & 1 \\
\hline Foeniculum vulgare Mill. & Apiaceae & 1 & 1 & 1 & 1 & 1 \\
\hline Fraxinus excelsior $\mathrm{L}$. & Oleaceae & & 1 & 1 & 1 & \\
\hline Glaucium corniculatum (L.) Curtis & Papaveraceae & 1 & 1 & 1 & 1 & \\
\hline Glycyrrhiza glabra L. & Fabaceae & 1 & 1 & 1 & 1 & 1 \\
\hline Gossypium herbaceum L. & Malvaceae & 1 & 1 & 1 & 1 & 1 \\
\hline Gratiola officinalis $\mathrm{L}$. & Plantaginaceae & & 1 & 1 & 1 & 1 \\
\hline Haloxylon persicum Bunge ex Boiss. \& Buhse & Amaranthaceae & & & 1 & & \\
\hline Hordeum distichon $\mathrm{L}$. & Poaceae & & & 1 & & \\
\hline Hordeum vulgare $\mathrm{L}$. & Poaceae & 1 & 1 & 1 & & \\
\hline Humulus lupulus L. & Cannabaceae & & & 1 & 1 & 1 \\
\hline Hyacinthus orientalis L. & Asparagaceae & & 1 & & & \\
\hline Hyoscyamus niger L. & Solanaceae & 1 & 1 & 1 & 1 & 1 \\
\hline Hypericum perforatum $\mathrm{L}$. & Hypericaceae & & 1 & 1 & 1 & 1 \\
\hline Hyssopus officinalis $\mathrm{L}$. & Lamiaceae & 1 & 1 & 1 & 1 & \\
\hline Indigofera tinctoria $\mathrm{L}$. & Fabaceae & & 1 & 1 & 1 & \\
\hline Inula helenium $\mathrm{L}$. & Asteraceae & & 1 & 1 & 1 & 1 \\
\hline Isatis tinctoria $\mathrm{L}$. & Brassicaceae & & 1 & 1 & & \\
\hline Juglans regia L. & Juglandaceae & 1 & 1 & 1 & 1 & 1 \\
\hline Lactuca sativa $\mathrm{L}$. & Asteraceae & & & 1 & 1 & \\
\hline Lactuca serriola L. & Asteraceae & 1 & 1 & 1 & 1 & \\
\hline Lagenaria siceraria (Molina) Standl. & Cucurbitaceae & & & 1 & & \\
\hline Lathyrus oleraceus Lam. & Leguminosae & & & 1 & 1 & \\
\hline Lemna minor $\mathrm{L}$. & Araceae & & 1 & 1 & 1 & \\
\hline Lepidium campestre (L.) W.T. Aiton & Brassicaceae & & & 1 & & \\
\hline Lepidium latifolium $\mathrm{L}$. & Brassicaceae & 1 & 1 & 1 & 1 & \\
\hline Lepidium perfoliatum $\mathrm{L}$. & Brassicaceae & & 1 & 1 & 1 & \\
\hline Lepidium sativum $\mathrm{L}$. & Brassicaceae & 1 & 1 & 1 & & \\
\hline Leptaleum filifolium (Willd.) DC. & Brassicaceae & & & 1 & & \\
\hline Lilium candidum $\mathrm{L}$. & Liliaceae & 1 & 1 & 1 & & \\
\hline Linum usitatissimum $\mathrm{L}$. & Linaceae & 1 & 1 & 1 & 1 & 1 \\
\hline Lithospermum officinale $\mathrm{L}$. & Boraginaceae & & & 1 & 1 & \\
\hline Lolium perenne $\mathrm{L}$. & Poaceae & & & 1 & & \\
\hline Lolium temulentum $\mathrm{L}$. & Poaceae & & 1 & 1 & & \\
\hline Lysimachia arvensis (L.) U. Manns \& Anderb. & Primulaceae & & 1 & 1 & 1 & \\
\hline Lysimachia vulgaris $\mathrm{L}$. & Primulaceae & & 1 & 1 & 1 & \\
\hline Malus domestica (Suckow) Borkh. & Rosaceae & 1 & 1 & 1 & & \\
\hline Malva sylvestris $\mathrm{L}$. & Malvaceae & 1 & & 1 & 1 & \\
\hline
\end{tabular}




\begin{tabular}{|c|c|c|c|c|c|c|}
\hline \multirow[t]{2}{*}{ Plant species } & \multirow[t]{2}{*}{ Family } & \multirow[t]{2}{*}{ Ar-Razi } & \multirow[t]{2}{*}{ Avicenna } & \multirow[t]{2}{*}{ Beruni } & \multicolumn{2}{|c|}{ Use in medicine } \\
\hline & & & & & Folk & Modern \\
\hline Marrubium vulgare $\mathrm{L}$. & Lamiaceae & & 1 & 1 & 1 & \\
\hline Medicago sativa $\mathrm{L}$. & Fabaceae & & 1 & 1 & & \\
\hline Melia azedarach L. & Meliaceae & & 1 & 1 & & \\
\hline Melilotus officinalis (L.) Lam. & Fabaceae & 1 & 1 & 1 & 1 & 1 \\
\hline Mentha $\times$ piperita $\mathrm{L}$. & Lamiaceae & & 1 & 1 & 1 & 1 \\
\hline Morus alba $\mathrm{L}$. & Moraceae & & 1 & 1 & 1 & 1 \\
\hline Morus nigra L. & Moraceae & & 1 & 1 & 1 & 1 \\
\hline Narcissus poeticus $\mathrm{L}$. & Amaryllidaceae & & 1 & 1 & & \\
\hline Narcissus tazetta $\mathrm{L}$. & Amaryllidaceae & & 1 & 1 & & \\
\hline Nasturtium officinale W.T. Aiton & Brassicaceae & & & 1 & 1 & \\
\hline Nepeta cataria L. & Lamiaceae & & & 1 & 1 & \\
\hline Nerium oleander $\mathrm{L}$. & Apocynaceae & & 1 & 1 & & 1 \\
\hline Nigella sativa L. & Ranunculaceae & & 1 & 1 & 1 & \\
\hline Nuphar lutea (L.) Sm. & Nymphaeaceae & & & 1 & 1 & 1 \\
\hline Nymphaea lutea L. & Nymphaeaceae & 1 & & 1 & & \\
\hline Ocimum basilicum $\mathrm{L}$. & Lamiaceae & 1 & 1 & 1 & 1 & \\
\hline Ononis spinosa subsp. antiquorum (L.) Briq. & Fabaceae & & & 1 & 1 & \\
\hline Onopordum acanthium $\mathrm{L}$. & Asteraceae & & 1 & 1 & 1 & 1 \\
\hline Oryza sativa $\mathrm{L}$. & Poaceae & & 1 & 1 & & \\
\hline Oxalis corniculata $\mathrm{L}$. & Oxalidaceae & & & 1 & & \\
\hline Panicum miliaceum $\mathrm{L}$. & Poaceae & 1 & 1 & 1 & & \\
\hline Papaver somniferum $\mathrm{L}$. & Papaveraceae & 1 & 1 & 1 & 1 & 1 \\
\hline Pastinaca sativa $\mathrm{L}$. & Apiaceae & & 1 & 1 & & \\
\hline Peganum harmala $\mathrm{L}$. & Nitrariaceae & & 1 & 1 & 1 & 1 \\
\hline $\begin{array}{l}\text { Pentanema britannicum (L.) D. Gut. Larr., } \\
\text { Santos-Vicente, }\end{array}$ & Asteraceae & & & 1 & 1 & \\
\hline Persicaria hydropiper (L.) Delarbre & Polygonaceae & & 1 & 1 & 1 & 1 \\
\hline Petroselinum crispum (Mill.) Nyman & Apiaceae & & 1 & 1 & 1 & 1 \\
\hline Phaseolus vulgaris $\mathrm{L}$. & Fabaceae & & 1 & 1 & 1 & 1 \\
\hline Phragmites australis (Cav.) Trin. ex Steud. & Poaceae & 1 & 1 & 1 & 1 & \\
\hline Picnomon acarna (L.) Cass. & Asteraceae & & 1 & 1 & 1 & \\
\hline Pimpinella anisum $\mathrm{L}$. & Apiaceae & 1 & 1 & 1 & 1 & 1 \\
\hline Pistacia vera $\mathrm{L}$. & Anacardiaceae & 1 & 1 & 1 & 1 & 1 \\
\hline Plantago major $\mathrm{L}$. & Plantaginaceae & & 1 & 1 & 1 & 1 \\
\hline Platanus orientalis $\mathrm{L}$. & Platanaceae & & 1 & 1 & & \\
\hline Polygonum aviculare $\mathrm{L}$. & Polygonaceae & & 1 & 1 & 1 & 1 \\
\hline Populus alba $\mathrm{L}$. & Salicaceae & & 1 & & & \\
\hline Populus nigra $\mathrm{L}$. & Salicaceae & & & 1 & 1 & \\
\hline Portulaca oleracea $\mathrm{L}$. & Portulacaceae & 1 & 1 & 1 & 1 & \\
\hline Potamogeton natans $\mathrm{L}$. & Potamogetonaceae & & 1 & 1 & 1 & \\
\hline Potentilla reptans $\mathrm{L}$. & Rosaceae & & 1 & 1 & 1 & \\
\hline Prunus armeniaca $\mathrm{L}$. & Rosaceae & 1 & 1 & 1 & 1 & 1 \\
\hline Prunus avium (L.) L. & Rosaceae & & & 1 & & \\
\hline Prunus cerasifera Ehrh. & Rosaceae & & & 1 & & \\
\hline Prunus cerasus L. & Rosaceae & & & 1 & 1 & 1 \\
\hline Prunus davidiana (Carrière) Franch. & Rosaceae & 1 & 1 & 1 & 1 & 1 \\
\hline Prunus domestica $\mathrm{L}$. & Rosaceae & 1 & 1 & 1 & 1 & 1 \\
\hline Prunus mahaleb L. & Rosaceae & & 1 & 1 & 1 & \\
\hline Prunus persica (L.) Batsch & Rosaceae & & 1 & 1 & 1 & \\
\hline Pseudohandelia umbellifera (Boiss.) Tzvelev & Asteraceae & & 1 & 1 & & \\
\hline Punica granatum $\mathrm{L}$. & Lythraceae & 1 & 1 & 1 & 1 & 1 \\
\hline Pyrus communis $\mathrm{L}$. & Rosaceae & 1 & 1 & 1 & 1 & \\
\hline Raphanus raphanistrum $\mathrm{L}$. & Brassicaceae & & & 1 & & \\
\hline Raphanus raphanistrum subsp. sativus (L.) & Brassicaceae & 1 & 1 & 1 & & \\
\hline \multicolumn{7}{|l|}{ Domin } \\
\hline Rhamnus cathartica L. & Rhamnaceae & 1 & & & & \\
\hline Rheum palmatum $\mathrm{L}$. & Polygonaceae & & 1 & 1 & 1 & 1 \\
\hline Rhus coriaria L. & Anacardiaceae & 1 & 1 & 1 & 1 & 1 \\
\hline Ricinus communis $\mathrm{L}$. & Euphorbiaceae & 1 & 1 & 1 & 1 & 1 \\
\hline Ridolfia segetum (L.) Moris & Apiaceae & 1 & 1 & 1 & 1 & 1 \\
\hline Rosa $\times$ damascena Herrm. & Rosaceae & & 1 & 1 & 1 & 1 \\
\hline Rosa alba L. & Rosaceae & & & 1 & 1 & 1 \\
\hline
\end{tabular}




\begin{tabular}{|c|c|c|c|c|c|c|}
\hline \multirow[t]{2}{*}{ Plant species } & \multirow[t]{2}{*}{ Family } & \multirow[t]{2}{*}{ Ar-Razi } & \multirow[t]{2}{*}{ Avicenna } & \multirow[t]{2}{*}{ Beruni } & \multicolumn{2}{|c|}{ Use in medicine } \\
\hline & & & & & Folk & Modern \\
\hline Rosa canina $\mathrm{L}$. & Rosaceae & & 1 & 1 & 1 & 1 \\
\hline Rubia tinctorum Salisb. & Rubiaceae & 1 & 1 & 1 & 1 & 1 \\
\hline Rumex acetosa $\mathrm{L}$. & Polygonaceae & 1 & 1 & 1 & 1 & \\
\hline Ruta graveolens L. & Rutaceae & 1 & 1 & 1 & & \\
\hline Salix aegyptiaca L. & Salicaceae & & 1 & 1 & & \\
\hline Salix alba L. & Salicaceae & & & 1 & & \\
\hline Salix babylonica L. & Salicaceae & & 1 & 1 & & \\
\hline Salix caprea $\mathrm{L}$. & Salicaceae & & 1 & 1 & & \\
\hline Sambucus nigra L. & Viburnaceae & & & 1 & & \\
\hline Senna alexandrina Mill. & Fabaceae & & 1 & 1 & 1 & 1 \\
\hline Senna alexandrina var. alexandrina & Fabaceae & 1 & 1 & 1 & 1 & 1 \\
\hline Senna italica Mill. & Fabaceae & & 1 & 1 & 1 & 1 \\
\hline Sesamum indicum $\mathrm{L}$. & Pedaliaceae & 1 & 1 & 1 & 1 & 1 \\
\hline Silybum marianum (L.) Gaertn. & Asteraceae & & 1 & 1 & & 1 \\
\hline Sinapis alba L. & Brassicaceae & & 1 & 1 & 1 & \\
\hline Sinapis arvensis $\mathrm{L}$. & Brassicaceae & & & 1 & & \\
\hline Sium sisarum $\mathrm{L}$. & Apiaceae & & 1 & 1 & & \\
\hline Solanum melongena $\mathrm{L}$. & Solanaceae & & 1 & 1 & & \\
\hline Solanum nigrum L. & Solanaceae & 1 & 1 & 1 & 1 & \\
\hline Sonchus oleraceus L. & Asteraceae & & & 1 & & \\
\hline Sorghum bicolor (L.) Moench & Poaceae & & & 1 & 1 & \\
\hline Spartium junceum L. & Fabaceae & & 1 & 1 & & \\
\hline Spinacia oleracea L. & Amaranthaceae & 1 & 1 & & 1 & \\
\hline Tamarix gallica L. & Tamaricaceae & 1 & & & & \\
\hline Tanacetum parthenium (L.) Sch. Bip. & Asteraceae & & 1 & 1 & 1 & \\
\hline Tanacetum parthenium (L.) Sch. Bip. & Asteraceae & & & 1 & & \\
\hline Taraxacum officinale F.H. Wigg. & Asteraceae & & 1 & 1 & 1 & 1 \\
\hline Taxus baccata L. & Taxaceae & & 1 & 1 & & \\
\hline Thymus serpyllum $\mathrm{L}$. & Lamiaceae & & 1 & 1 & 1 & 1 \\
\hline Trachyspermum ammi(L.) Sprague & Apiaceae & & & 1 & 1 & \\
\hline Tribulus terrestris $\mathrm{L}$. & Zygophyllaceae & 1 & 1 & 1 & 1 & 1 \\
\hline Trifolium fragiferum $\mathrm{L}$. & Fabaceae & & & 1 & 1 & \\
\hline Trifolium pratense $\mathrm{L}$. & Fabaceae & & 1 & & & \\
\hline Trigonella foenum-graecum $\mathrm{L}$. & Fabaceae & 1 & 1 & 1 & 1 & \\
\hline Triticum aestivum $\mathrm{L}$. & Poaceae & 1 & 1 & 1 & 1 & \\
\hline Tussilago farfara $\mathrm{L}$. & Asteraceae & & 1 & 1 & 1 & 1 \\
\hline Typha latifolia L. & Typhaceae & & 1 & 1 & 1 & \\
\hline Ulmus minor Mill. & Ulmaceae & & & 1 & & \\
\hline Urtica dioica $\mathrm{L}$. & Urticaceae & 1 & 1 & 1 & 1 & 1 \\
\hline Verbascum songaricum Schrenk & Scrophulariaceae & & 1 & 1 & 1 & 1 \\
\hline Verbena officinalis $\mathrm{L}$. & Verbenaceae & & 1 & 1 & 1 & \\
\hline Vicia ervilia Willd. & Fabaceae & 1 & 1 & 1 & & \\
\hline Vicia faba $\mathrm{L}$. & Fabaceae & 1 & 1 & 1 & & \\
\hline Vicia lens (L.) Coss. \& Germ. & Fabaceae & 1 & 1 & 1 & 1 & \\
\hline Vicia sativa $\mathrm{L}$. & Fabaceae & & 1 & 1 & & \\
\hline Viola odorata $\mathrm{L}$. & Violaceae & 1 & & 1 & 1 & \\
\hline Vitex agnus-castus $\mathrm{L}$. & Lamiaceae & & 1 & 1 & 1 & \\
\hline Vitis vinifera $\mathrm{L}$. & Vitaceae & 1 & 1 & 1 & 1 & 1 \\
\hline Ziziphus jujuba Mill. & Rhamnaceae & & 1 & 1 & 1 & \\
\hline Total & & 87 & 182 & 231 & 173 & 86 \\
\hline
\end{tabular}

Medicinal herbs mostly belong to the families of Asteraceae, Fabaceae, Rosaceae, Brassicaceae, Apiaceae, Poaceae and other (Fig. 2).

Our analysis of the references showed that Prunus (8 species), Brassica (4), Lepidium (4), Rosa (3), Salix (4), Vicia (4), Allium (3), Amaranthus (3), Artemisia (3), Cucumis (3), and Senna (3) were the dominant medicinally used plant genera. Let us consider in more detail the genus Prunus, which is most of the species in this genus (Table 3). 


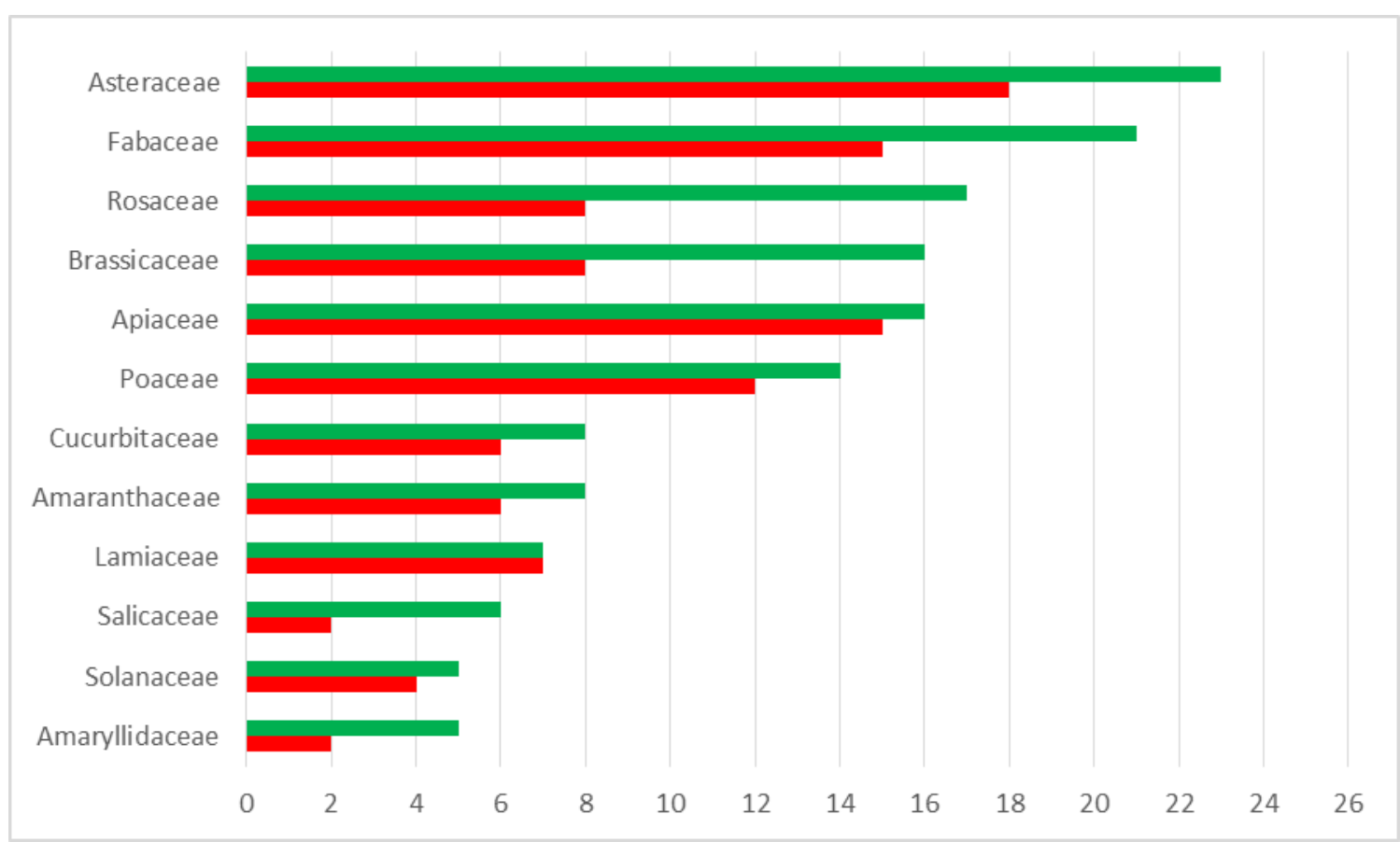

Figure 2. Number of genera (red) and species (green) in the ten most cited plant families.

Nowadays, among the plants used and cited in the writings of the named scientists of the Middle East, more than 50 species of plants are included in official herbal medicine directories (Muravyova 1964; Wolf, Maleeva 1969; Sokolov, Zamotaev 1984) and pharmacopeias (Obomaikova, Arzamastsev 1968). These are the following taxa: Acorus calamus L., Aloe vera L., Pimpinella anisum L., Althaea officinalis L., Artemisia absinthium L., A. cina O. Berg., A. vulgaris L., Calendula officinalis L., Senna alexandrina Mill., Citrullus colocynthis (L.) Schrad., Commiphora kua (R.Br. ex Royle) Vollesen, Crataegus sanguinea Pall., Datura stramonium L., Drimia maritima (L.) Stearn, Dryopteris filix-mas (L.) Schott, Equisetum arvense L., Euphorbia resinifera O. Berg, Hyoscyamus niger L., Hypericum perforatum L., Inula helenium L., Juniperus communis L., Glycyrrhiza glabra L., Linum usitatissimum L., Matricaria chamomilla L., Melilotus officinalis (L.) Lam., Mentha $x$ piperita L., Origanum vulgare L., Papaver somniferum L., Persicaria hydropiper (L.) Delarbre, Peganum harmala L., Prunus amygdalus Batsch, Punica granatum L., Quercus robur L., Rhamnus cathartica L., Rheum officinale Baill., R. palmatum L., Ricinus communis L., Rosa beggeriana Schrenk, $R$. fedtschenkoana Regel, Salvia officinalis L., Sinapis alba L., Thymus serpyllum L., Tussilago farfara L., Trachyspermum ammi (L.) Sprague, Taraxacum officinale F.H. Wigg., Urtica dioica L., Vachellia nilotica subsp. tomentosa (Benth.) Kyal. \& Boatwr., Valeriana officinalis L. and others.

According to Khalmatov (Khalmatov 1958, 1984, 1999) and (Khojimatov et al. 1995), the number of medicinal plants in the flora of Uzbekistan exceeds 1157 species, and by the definition of Khojimatov (Khojimatov 2021), their number is even more than 1200. According to the results of our study, among the medicinal plants currently used in folk and scientific medicine, more than 240 species were cited in the works of Ar-Razi, Avicenna, Beruni, and Yusufi. In this article we only provide Avicenna data on some of the medicinal plants of Uzbekistan currently often used in folk medicine.

Acorus calamus L. - plant juice applied as a diuretic and for gastrointestinal pain. In folk medicine, infusion from rhizomes is used as a tonic and expectorant for coughing and chest pains.

Althaea officinalis L. - decoction from roots, seeds and leaves was recommended for inflammation of the lungs, pleurisy and as an expectorant. In folk medicine, roots, leaves, and flowers are used for coughing and chest pains Artemisia spp. - recommended a bath from the aboveground part of wormwood in the treatment of renal stone disease. He used a decoction of the herb for the common cold and as a biliary and diuretic biliary. In folk medicine, flower decoction is used as biliary, sweating, clay and antipyretic.

Glycyrrhiza glabra L. - Avicenna used the roots of the plant for kidney ulcers and inflammation of the stomach, fever, and pulmonary diseases. In folk medicine, decoction and root extract are used as sweating and laxative agents. Hyoscyamus niger L. - squeezed leaf juice in the ear, eye, and dental pain. In folk medicine, a leaf patch is applied to draw pus and used as an analgesic. 
Table 3. Selected list of economically important and medicinal plant species used in folk and modern medicine.

\begin{tabular}{|c|c|c|c|c|}
\hline Scientific name & $\begin{array}{l}\text { Local } \\
\text { name }\end{array}$ & Parts used & Used to folk medicine & Used for \\
\hline $\begin{array}{l}\text { Prunus avium (L.) } \\
\text { L. }\end{array}$ & Gilos & $\begin{array}{l}\text { Fruits, } \\
\text { fruit stalks }\end{array}$ & $\begin{array}{l}\text { Cherry juice is used as an expectorant for bronchitis and tracheitis, an } \\
\text { aqueous infusion of fruit pulp is a refreshing and antipyretic agent for } \\
\text { colds }\end{array}$ & $\begin{array}{l}\text { The plant emits a lot of gum, which is used in } \\
\text { textile production and in the finishing of fabrics. } \\
\text { The bark contains } 7-10 \% \text { tannins, which allows it } \\
\text { to be used for tanning leather. Bark and roots } \\
\text { used to be used for dyeing wool and fabrics. The } \\
\text { wood is suitable for joinery, hoops are made } \\
\text { from young trunks. Cherry pipes and } \\
\text { mouthpieces are well-known. Melliferous. }\end{array}$ \\
\hline Prunus cerasus L. & Olcha & $\begin{array}{l}\text { Fruits, } \\
\text { fruit stalks, } \\
\text { bark }\end{array}$ & $\begin{array}{l}\text { The influence of cherries and cherry juice on the heart and blood vessels } \\
\text { is known. Vitamin PP together with ascorbic acid has a positive effect on } \\
\text { blood vessels, increasing their permeability. And the coumarins in the } \\
\text { berry are involved in regulating the clotting process, preventing the } \\
\text { formation of blood clots and thinning the blood. }\end{array}$ & $\begin{array}{l}\text { Food, melliferous, technical, decorative. Used in } \\
\text { cosmetology. }\end{array}$ \\
\hline $\begin{array}{l}\text { Prunus cerasifera } \\
\text { Ehrh. }\end{array}$ & Togolcha & $\begin{array}{l}\text { Flowers, } \\
\text { leaves, } \\
\text { fruits }\end{array}$ & $\begin{array}{l}\text { Cherry plum leaves are often used because of the high content of active } \\
\text { ingredients in them and the possibility of long-term storage. The leaves } \\
\text { are added to herbal mixtures - collections and herbal teas, and they are } \\
\text { also brewed on their own for the following diseases: coughs, colds; } \\
\text { vitamin deficiency, anemia; as diaphoretic, cleansing agent; diseases of } \\
\text { the gastrointestinal tract; diseases of the kidneys and liver; impotence } \\
\text { Tea made from cherry plum leaves is recommended to be included in } \\
\text { their diet for pregnant and lactating women, as well as for older people } \\
\text { and children, since cherry plum is simply an irreplaceable vitamin remedy } \\
\text { in the autumn-winter period. The infusion of cherry plum leaves has a } \\
\text { relaxing and calming effect on humans. It has a very beneficial effect on } \\
\text { the functioning of the central nervous system. Helps with stress. Cherry } \\
\text { plum is also an excellent laxative and anti-scing agent. }\end{array}$ & Food, melliferous, technical, decorative. \\
\hline $\begin{array}{l}\text { Prunus } \\
\text { domestica L. }\end{array}$ & Olcha & $\begin{array}{l}\text { Flowers, } \\
\text { leaves, } \\
\text { fruits, bark }\end{array}$ & $\begin{array}{l}\text { Dried plum fruits, which, due to their high fiber content, are able to } \\
\text { enhance intestinal motility. Due to the presence of pectins, plums have } \\
\text { the ability to remove radionuclides from the body. The fruits of the plant } \\
\text { are popularly used in the treatment of anemia, hypertension, } \\
\text { rheumatism, chronic constipation, diseases of the organs of the excretory } \\
\text { system. Freshly squeezed plum juice is also a dietary product that } \\
\text { improves the functioning of the gastrointestinal tract. }\end{array}$ & $\begin{array}{l}\text { Food, melliferous, ornamental. Fatty oil from } \\
\text { plum seeds, obtained by cold pressing, is used } \\
\text { for pharmaceutical and cosmetic purposes along } \\
\text { with almond oil. Plum fruits are widely used in } \\
\text { food, confectionery and alcoholic beverage } \\
\text { industries. }\end{array}$ \\
\hline $\begin{array}{l}\text { Prunus persica } \\
\text { (L.) Batsch }\end{array}$ & Shaftoli & $\begin{array}{l}\text { Flowers, } \\
\text { leaves, } \\
\text { fruits, bark }\end{array}$ & $\begin{array}{l}\text { Peach fruits are prescribed for dietary nutrition of patients with anemia, } \\
\text { heart disease, as a diuretic, adaptogenic agent. Peaches, due to the } \\
\text { presence of phenolic compounds, flavonoids, have antioxidant } \\
\text { properties, especially the peel of the fruit. The bark of the peach tree } \\
\text { prevents the development of benign prostatic hyperplasia. }\end{array}$ & $\begin{array}{l}\text { Peach seed oil is used as a solvent for medicinal } \\
\text { substances, along with plum and apricot seed oil. }\end{array}$ \\
\hline
\end{tabular}


Inula helenium L. - used grass for inflammation of the sciatic nerve, for joint pains, and root syrup as a diuretic. In folk medicine, infusion and decoction from roots are used as an expectorant, diuretic, antipyretic agent.

Punica granatum L. - flowers were used to treat gastric diseases, bleeding gums, and fruits as a diuretic and astringent agent in gastric diseases. In ethnomedicine, it is used as a painkiller, a clay agent, as well as in scabies, jaundice, and intestinal infections.

Trachyspermum copticum Link. - Avicenna recommended an infusion of fruits as a diuretic, to cleanse the kidneys and urinary bladder. In folk medicine, it is used as a clay and gastric agent.

Tussilago farfara L. - used as an expectorant for coughing, fresh leaf juice - as a biliary and diuretic. In ethnomedicine, they are used for the same diseases.

Taraxacum officinale F.H. Wigg. - juice from fresh leaves was recommended for watering and blockage of the liver. In folk medicine, fresh leaf juice is recommended for chest pains and as a laxative.

\section{The use of medicinal fungi}

Considering the total estimated number of fungal species (about 5.1 million), it is no surprise that among them are species that produce important compounds (e.g., penicillin) that form the basis of several classes of medicinal products, such as antibiotics and immune enhancers, as well as some species that are a danger to human health (e.g., Aspergillus and Candida species).

Mushrooms, such as reishi (also known as ling zhi; Ganoderma lucidum, Ganodermataceae), have been used medicinally in Asia for centuries (Wasser 2005; McMeekin 2005). Complementing this rich history of use, numerous scientific studies have been performed on mushroom extracts for their potential health benefits. Many fungi contain a rich enzyme kit and physiologically active substances. These properties are widely used by humans. The discovery of antibiotics is one of the recent achievements. Penicillin, a product of the life of Penicillium fungi, was widely used in Medicine. Using the fungus Aspergillus niger, citric acid is obtained, gibberellin from the marsupial stage of Gibberella fujikuroi is used as a growth substance (Brain et al. 1954). It is well known that some species of fungi are widely used by the population as food and therefore even they are specially bred. Although the mycobiota of the Central Asian region have been explored by different mycologists since the 20th century, the diversity of fungi there have largely been neglected, resulting in a very fragmentary knowledge of this resource in the region (Gafforov 2017; Gafforov and Rakhimova 2017; Gafforov et al. 2016, 2017, 2019; Cheek et al. 2020; Abdurazakov et al. 2021; Wang et al. 2021; Lestari et al. 2021; Htet et al. 2021). In Uzbekistan, medicinal and edible fungi are particularly poorly studied and not exploited at all (Gafforov 2014; Yatsiuk et al. 2016; Gafforov et al. 2020, Wang et al. 2021).

In the East, mushrooms have been known as a medicine for centuries. In the works of Ar-Razi (Clinical records of Abu Bakr Razii 1994; Ar-Razi 1994), Avicenna (Ibn Sina 1996) and Beruni (Beruni 1973), both the benefit and the harm of mushrooms are mentioned. For example:

Some biological organisms have been mentioned by the Holy Qura'n and Sahih al-Bukhari, including mushrooms such as truffles and are known under the names "Kama'ah" and "Fagaa" in Arabic (Khalifa et al. 2019). Taxonomically, truffles are ascomycetes fungi belonging to several genera are in six Glaziellaceae, Discinaceae, Morchellaceae, Helvellaceae, Tuberaceae, Pezizaceae, and Pyronemataceae families. Such as one of important edible and medicinal genus Tuber, the family Tuberaceae, and the order Pezizales, contains at least ca. 230 species worldwide, associated with ectomycorrhizal forms and the roots of a broad range of both gymnosperm and angiosperm hosts spread over the temperate forests, flood plains, and subtropical forests habitats (O'Donnell et al. 1997; Benucci et al. 2011, 2012a, $2012 b)$. Truffle is highly appreciated as a dietary component and nutritional substitute and the great consumption is owned to its high aroma taste and musky flavor (Al-Laith 2010; Dahham et al. 2018; Feng et al. 2019; Mustafa et al. 2020). Furthermore, truffle oil, used in different types of salads, contains a broad range of volatile organic compounds (Wernig et al. 2018). Truffles are enriched in a wide range of bioactive chemical constituents such as anthocyanins, oligosaccharides, flavonoids, carotenoids, pheromones, and steroids. In addition to being used as food all over the world, truffles are also valued as therapeutic agents with antioxidant, anti-mutagenic, anticarcinogenic, anti-microbial, anti-viral, immune-modulating, anti-inflammatory, anti-depressant (El Enshasy et al., 2013). With the high price and rarity of truffle fungi, they are highly vulnerable to fraud, as in the case of Tuber melanosporum, the "black truffle", which is referred to as the black diamond of cuisine and is considered one of the most culinary and commercially valued species of truffles. (Feng et al. 2019; Mustafa et al. 2020). Tuber melanosporum (called "Al-Kamaa" or "Al-Fag'a" or "Kama" in Arabic), is given that the best sandy, white, they must be boiled in water with salt, peeled and cut in half, boiled in olive oil with spices and gum stink ferules (Ferula assafoetida L.). When poisoned with it, you need to take pure wine with spices. Mushrooms cause paralysis of the joint, 
and "sacta" disease (apollection) and make urination difficult. Avicenna described the truffles as a cure in the 10th century, for a variety of disorders like vomiting, wounds, and weakness. In one of his famous books namely the "AlQanun fi't-Tibb" (The Canon of Medicine) written in the tenth century as part of the Arabian Traditional Medicin. It was used as a medical textbook in the Islamic world and Europe up to the 18th century. Avicenna recommended desert truffle juice used for common eye inflammations.

Curly ("Gushna," "Futr"), or Morchella esculenta shrivels like cartilage, its shape is like a small, shriveled cup. They wash their dress and eat with sour condiments. By Beruni, the curly ("Gushana") is also mentioned as a laundry detergent. When dried, the curls become white and inside red. They are put in an antimony vessel, they have the color of dust with a black tint and are a medicine for the eyes, applying such an ointment with a stick. Morels, a group of the world's highly valued edible and medicinal mushrooms, are of very important economic and scientific value. Most morel mushrooms contain a wide range of active constituents which include tocopherols, carotenoids, organic acids, polysaccharides, and phenolic compounds which exhibit a wide range of medicinal and pharmacological properties including anti-microbial, anti-inflammatory, immunostimulatory, antitumor, and antioxidant (Du et al. 2015; Liu et al. 2018). It is also used for the treatment of indigestion, excessive phlegm, and asthma (Tietel et al. 2018).

Agarikon or Gharikon and scientific name is Fomitopsis officinalis (Vill.) Bondartsev \& Singer in appearance "dense, like wood, yellow, smooth in the places of breaking, sweet in taste, means good." This species is commonly known as the Agarikon or Gharikon mushroom in Iran (Vazirian et al., 2016). Fomitopsis officinalis is a wood-rotting fungus that found on various conifer trees. The fungus is native to Europe, Asia, and North America. Agarikon has been in use for thousands of years due to its healing potential and it was originally found by Greek physician Dioscorides Pedanios in the 1st century AD. This fungal species is a treatment against consumption illnesses such as pulmonary tuberculosis. The basidiome of Agarikon has also been carved to make masks used by Native Indians used during rituals, indicating the size of this mushroom. In modern medicine, Fomitopsis officinalis used as a source of an antibacterial, antifungal, antiviral, anti-inflammatory well as and immunostimulant and antitumor agents (Lindequist et al. 1990; Blagodatsji et al. 2018; Elkhateeb et al. 2019).

Some other species of truffles (in Arabic "Facwat-dabu," "Bondage") are like a stick with a head. They are boiled when the mushrooms dry and bear fruit, or at the very beginning of growth. In the paragraphs on fungi, Beruni, referring to the work of other authors, leads to the benefit and harm of mushrooms: for example, Indian troops who welded and ate harmful mushrooms were poisoned, resulting in the death of 13 people. The species of fungi, called "Nulita" (i.e., Boletus) was considered the best, and other "Amanita," on the contrary, are dangerous to life. On killing mushrooms there is sticky moisture, after collection, they quickly rot and spoil. In the paragraph "Futr" (mushrooms) there are references to Dioscorides and Galen, which also indicated the benefit and harm of mushrooms.

Medieval sources also state that mushrooms that appear in the vipers and snake's nests or grow under olive (Olea europaea L.) and in places where there is the dung of donkeys and other horse-like animals are called "snake mushrooms," because they are deadly toxic.

This data, written almost 1000 years ago, has not lost significance to this day. The people of Central Asia have long distinguished edible and harmful mushrooms when used.

\section{Conclusion}

The science of medicine in the ancient East has a deep connection to the founders of the Greek medical school, e.g., Hippocrates, Aristotle, Dioscorides, Galen, etc. Greek folk medicine was later adopted in the East - in Syria and Persia. There was even a medical school in Persia, where ancient Greek books on medicine were translated into Arabic. Scientists-physicians of the medieval East like Razi, Avicenna, Beruniy and others also belonged to that school. The Arabian pharmacopeia widely used complex recipes, which contained various herbs. In Arab countries, special books ("Karabadins") describing the methods of preparation and consumption of medicines were written. Later these books were then translated in neighboring Muslim states and Europe. It should be noted that the comprehensive medieval knowledge still will provide an opportunity to discover new medicinal plants and remedies, as well as help to introduce them into practical medicine. We will continue this work in the future and provide more information on the use of medicinal plants and fungi in folk and modern medicine. 
A book of Ar-Razi, "Clinical Records of Abu Bakr Razi and His Disciple," describes the histories of more than 750 diseases and methods of treating them with medicinal herbs. About 173 medicinal plants used by Ar-Razi were mentioned more than a century later in the writings of Avicenna and Beruni. The book of "The Canon of Medicine" (Al-Konun) written by Avicenna consists of 5 volumes. The drugs are mainly described in the second volume "Medicinal Plants" which contains about 515 plants names. In Beruni's book of Saydana (Kitab as-Saidana fit-tib), the names of 901 medicinal plants, 107 minerals, and 101 animal products are given in different languages. Beruni was one of the first to compile a dictionary of medicinal plants, citing the names of medicines in various languages of the East at that time, including Central Asia. Yusufi, Quatrains (Rubayat), described the healing properties of more than 110 plants and the methods of preparation of medicines.

In conclusion, this study provides the first list of medicinal plants and some fungi with new names of plant species and other ethnobotanical information from the medieval east. These species are economically important as sources of food and medicines. Beyond local scale, these data are also crucial as a supplement of the worldwide knowledge of ethnobiology for elucidating folk and scientific medicine.

\section{Declarations}

List of abbreviations: Not applicable.

Ethics approval and consent to participate: Not applicable.

Consent for publication: Not applicable.

Availability of data and materials: All the data are presented in tables in the manuscript and are available with the corresponding authors.

Competing interests: The authors declare that they have no competing interests.

Funding: Ministry of Innovative Development of the Republic of Uzbekistan supported these research projects (Nos. A-FA-2021-144 and MUK-2021-46).

Authors' contributions: KT, YG collected, analyzed the data and drafted the manuscript. YG, OK, TM, RB and NN advised, reviewed, and approved the final manuscript.

\section{Acknowledgements}

This paper is part of our research projects that were hosted financially and materially supported by the Ministry of Innovative Development of the Republic of Uzbekistan. We are also indebted to thank the anonymous reviewer for reading the manuscript and providing important comments. YG thanks Dr. Khusniddin Mamadaliev (Institute of History, Uzbekistan Academy of Sciences) for his communication about the history of the medieval east.

\section{Literature Cited}

Abu Ali Ibn Sino. Canon of Medicine. 1996. Tashkent: Vol. 2. 336 p.

Abu Bakr Ar-Razi. Clinical records. 1994. Tashkent: 251. (In Uzbek)

Abu Bakr Razii, Clinical records of Abu Bakr Razii, his student. 1973 Fan Publishing House. 335. (in Uzbek).

Abu Raikhan Beruni. Selected works, V. 4. 1973. Fan Publishing House. 1120. (in Russian)

Al-Laith AAA. 2010. Antioxidant components and antioxidant/antiradical activities of desert truffle (Tirmania nivea) from various Middle Eastern origins. Journal of Food Composition and Analysis 23:15-22. doi: 10.1016/J.JFCA.2009.07.005

Amjad MS, Arshad M, Qureshi R. 2015. Ethnomedicinal inventory and folk uses of indigenous plants from Pir Nasoora National Park, Azad Jammu and Kashmir. Asian Pac J Trop Biomed 5:234-41.

Angiosperm Phylogeny Group 2016. An update of the Angiosperm Phylogeny Group classification for the orders and families of flowering plants: APG IV. Botanical Journal of the Linnean Society 181(1):1-20, doi:10.1111/boj.12385.

Arzumetov YS, Yuldashev KY 1983. Ibn Sino's medical views. Tashkent: Medicine Publishing House. 120

Azaizeh H, Saad B, Khalil K, Said O. 2006. The state of the art of traditional arab herbal medicine in the eastern region of the Mediterranean: a review. Evidence-Based Complementary and Alternative Medicine 3(2):229-35. doi: 10.1093/ecam/nel034. 
Benucci GMN, Bonito G, Falini L, Bencivenga M. 2012b. Mycorrhization of Pecan trees (Carya illinoinensis) with commercial truffle species: Tuber aestivum Vittad and Tuber borchii Vittad. Mycorrhiza 22:383-392. doi: 10.1007/s00572-011-0413-z

Benucci GMN, Gogan Csorbai A, Di Massimo G, Baciarelli Falini L, Bencivenga M, Donnini D. 2012a. Mycorrhization of Quercus robur L. and Corylus avellana L. seedlings with Tuber macrosporum Vittad. Mycorrhiza. doi: $10.1007 /$ s00572-012-0441-3

Benucci GMN, Raggi L, Albertini E, Grebenc T, Bencivenga M, Falcinelli M, Di Massimo G. 2011. Ectomycorrhizal communities in a productive Tuber aestivum Vittad. orchard: composition, host influence and species replacement. FEMS Microbiology Ecology 76(1):170-184. doi: 10.1111/j.1574-6941.2010.01039.x

Blagodatski A, Yatsunskaya M, Mikhailova V, Tiasto V, Kagansky A,Katanaev VL. 2018. Medicinal mushrooms as an attractive new source of natural compounds for future cancer therapy. Oncotarget 9:29259-29274. doi: 10.18632/oncotarget.25660

Brian PW, Elson GW, Hemming HG, Radley M. 1954. The plant-growth-promoting properties of gibberellic acid, a metabolic product of the fungus gibberella fujikuroi. Journal of the Science of Food and Agriculture 5(12):602-612. doi: $10.1002 /$ jsfa.2740051210.

Burghart DL, Sabonis-Helf T (eds). 2018. Central Asia in the era of sovereignty: the return of Tamerlane? Lanham, Lexington Books, p. 521.

Cheek M, Nic Lughadha E, Kirk P, Lindon H, Carretero J, Looney B, Douglas B, Haelewaters ID, Gaya E, Llewellyn T. et al. 2020. New discoveries: Plants and Fungi. Plants, People, Planet 2:371-388. doi: 10.1002/ppp3.10148

Cherepanov SK. 1995. Vascular Plants of the USSR. Sanct Petersburg, Mir i semiya Publishing House, 992 (In Russian).

Dahhama SS, Al-Rawia SS, Ibrahim AH, Abdul Majid AS, Abdul Majida AMS. 2018. Antioxidant, anticancer, apoptosis properties and chemical composition of black truffle Terfezia claveryi. Saudi Journal of Biological Sciences 25(8):1524-1534. doi: 10.1016/j.sjbs.2016.01.031

Du XH, Zhao Q, Yang ZL. 2015. A review on research advances, issues, and perspectives of morels. Mycology 96(2):78-85. doi: 10.1080/21501203.2015.1016561

El Enshasy H, Elsayed EA, Aziz R, Wadaan MA. 2013. Mushrooms and Truffles: Historical Biofactories for Complementary Medicine in Africa and in the Middle East. Evidence-Based Complementary and Alternative Medicine 2013:620451. doi: 10.1155/2013/620451

Elkhateeba WA, Dabaa GM, Elnahasa MO, Thomasb PW. 2019. Fomitopsis officinalis mushroom: ancient gold mine of functional components and biological activities for modern medicine. Egyptian Pharmaceutical Journal 18(4):285289. doi: 10.4103/epj.epj_46_19

Feng T, Shui M, Song S, Zhuang H, Sun M, Yao L. 2019. Characterization of the Key Aroma Compounds in Three True Varieties from China by Flavoromics Approach. Molecules 24(18):3305. doi: 10.3390/molecules24183305

Flora of Uzbekistan. 1941-1962. Vol. 1-6. Fan Publishing House.

Gafforov Y, Abdurazzokov A, Yarasheva M, Ono Y. 2016. Rust fungi from the Fergana Valley, Chatkal and Kurama Mountain Ranges in Uzbekistan. Stapfia Reports 105:161-175.

Gafforov Y, Ordynets A, Langer E, Yarasheva M, Gugliotta AM, Schigel D, Pecoraro L, Zhou Y, Cai L, Zhou LW. 2020. Species diversity with comprehensive annotations of woodinhabiting poroid and corticioid fungi in Uzbekistan. Frontiers in Microbiology 11:598321. doi: 10.3389/fmicb.2020.598321

Gafforov Y, Phookamsak R, Jiang HB, Wanasinghed DN, Juliev M. 2019. Ophiobolus hydei sp. nov. (Phaeosphaeriaceae, Ascomycota) from Cirsium and Phlomoides in Uzbekistan. Botany 97:671-680. doi: 10.1139/cjb-2019-0118

Gafforov Y, Rakhimov D. 2017. Diplodia and Dothiorella species (Botryosphaeriaceae, Ascomycota) from Uzbekistan. Journal of the Botanical Research Institute of Texas 11(2):455-467.

Gafforov Y, Riebesehl J, Ordynets A, Langer E, Yarasheva M, Ghobad-Nejhad M, Zhou LW, Wang XW, Gugliota AM. 2017. Hyphodontia (Hymenochaetales, Basidiomycota) and similar taxa from Central Asia. Botany 95:1041-1056. doi: 10.1139/cjb-2017-0115 
Gafforov Y. 2017. A preliminary checklist of ascomycetous microfungi from Southern Uzbekistan. Mycosphere 8(4):660-696. doi: 10.5943/mycosphere/8/4/12

Howes MJ, Quave CL, Collemare J, Tatsis EC, Twilley D, Lulekal E, Farlow A, Li L, Cazar ME, Leaman D J, Prescott TAK, Milliken W, Martin C, De Canha MN, Lall N, Qin H, Walker BE, Vásquez-Londoño C, Allkin B, et al. 2020. Molecules from nature: Reconciling biodiversity conservation and global healthcare imperatives for sustainable use of medicinal plants and fungi. Plants, People, Planet 2: 463-481. doi.org/10.1002/ppp3.10138

Htet ZH, Mapook A, Gafforov Y, Chethana KWT, Lumyoung S, Hyde KD. 2021. Molecular phylogeny and diversity of Laburnicola (Didymosphaeriaceae): a new species from Uzbekistan. Phytotaxa 527 (3):177-190. doi.org/10.11646/phytotaxa.527.3.2

Index Fungorum. http://indexfungorum.org/ (accessed: 04.10.2021)

Iwu MM. 1994. African medicinal plants in the search for new drugs based on ethnomedicinal leads. In: Chadwick DJ, Marsh J, editors. Ethnobotany and the search for new drugs, Ciba foundation symposium 185. Chichester: Wiley; $116-29$.

Karimov UI. 1973. Manuscript "Saidana" and the history of its study. Beruni. Selected works. V.4. Tashkent, 13-34.

Keys to plants of Central Asia. Tashkent: Fan. 1968-1993. T. 1-10.

Khalifaa SAM, Farag MA, Yosrie N, Sabirf JSM, Saeedh A, Al-Mousawii SM, Tahaj W, Musharraf SG, Patell S, El-Seedi HR. 2019. Truffles: From Islamic culture to chemistry, pharmacology, and food trends in recent times. Trends in Food Science \& Technology 91:193-218. doi: 10.1016/j.tifs.2019.07.008

Khalmatov KhKh. 1958. To the study of medicinal plants described in the "Canon" of Abu Ali Ibn Sina (1st communication). Medicine Journal of Uzbekistan 7:65-72.

Khalmatov KhKh. 1999. Problems of systematization of plants, given in the "Canon of Medicine" by lbn Sino. Chemistry and Pharmacognosy 6:10-14.

Khasani M. 1992. Attending physician Babur-Yusufi. Translation and commentary by M. Khasani. Fan Publishing House. 190. (in Uzbek)

Khojimatov Kh.Kh., Aprasidi GS, Khojimatov OK. 1995. Wild medicinal plants of Central Asia. Abu Ali Ibn Sina Publishing House. 112.

Khojimatov O, Shagiakhmetova G, Shulgina N. 2018. Sixth National Report of the Republic of Uzbekistan on the conservation of biological diversity. United Nations Development Programme (UNDP). UNDP National Report No 6, 207.Kholmatov Kh.Kh. 1984. Wild medicinal plants of Uzbekistan. Medicine Publishing House. 277. (in Uzbek).

Khojimatov OK, Khamraeva DT, Khujanov AN, Bussmann RW. 2020. An overview of Ethnomedicinal plants of Uzbekistan. Ethnobotany Research \& Applications 20:08; doi.org/10.32859/era.20.08.1-19.

Khojimatov OK. 2021. Medicinal plants of Uzbekistan (properties, application and rational use). Manaviyat Publishing House. 328. (in Russian).

Kuchkarov B, Sherimbetov K, Aripdzhanov M, Gabitova R, Mitropolskaya J, Sobirov U, Talskikh V,

Lestari AS, Wanasinghe DN, Gafforov Y, Tennakoon DS, Chethana KWT, Aburazakov A, Promputtha I, Hyde KD. 2021. Taxonomy and phylogenetic appraisal of Leptosphaeria chatkalica sp. nov. (Leptosphaeriaceae, Pleosporales) from Uzbekistan. Phytotaxa. 520(2). doi: 10.11646/phytotaxa.520.2.3. 155-168

Lindequist U, Teuscher E, Narbe G. 1990. New active ingredients from Basidiomycetes. Rational Phytotherapy 11:139-149.

Liu Q, Ma H, Zhang Y, Dong C. 2018. Artificial cultivation of true morels: current state, issues and perspectives. Critical Reviews in Biotechnology 38(2):259-271. doi: 10.1080/07388551.2017.1333082.

Lubin N. 1984. Labour and Nationality in Soviet Central Asia: An Uneasy Compromise. Palgrave Macmillan. 305. https://doi.org/10.1007/978-1-349-07204-0

Malik ZA, Bhat JA, Ballabha R, Bussmann RW, Bhatt AB. 2015. Ethnomedicinal plants traditionally used in health care practices by inhabitants of Western Himalaya. Journal of Ethnopharmacology 172:133-144. 
McMeekin D. 2005. The perception of Ganoderma lucidum in Chinese and Western culture. Mycologist 18:165-169. doi: 10.1017/S0269-915X(04)00406-9

Muravyova DA. 1964. Tropical medicinal plants. Medicine Publishing House. 462. (in Russian).

Mustafa AM, Angeloni S, Nzekoue FK, Abouelenein D, Sagratini G, Caprioli G, Torregiani E. 2020. An Overview on Trufflee Aroma and Main Volatile Compounds. Molecules 25:5948; doi:10.3390/molecules25245948

O'Donnell K, Cigelnik E, Weber NS, Trappe JM. 1997. Phylogenetic relationships among ascomycetons truffles and the true and false morels inferred from $18 \mathrm{~S}$ and $28 \mathrm{~S}$ ribosomal DNA sequence analysis. Mycologia 89(1):48-65.

Obomaikova AN, Arzamastsev AP. (ed.) 1968. State Pharmacopoeia of the USSR. Medicine Publishing House. 1078 (in Russian).

Plants of the World Online. http://www.plantsoftheworldonline.org/ (accessed 07.09.2021).

Raj AJ, Biswakarma S, Pala NA, Shukla G, Kumar M, Sumit Chakravarty S, Bussmann RW. 2018. Indigenous uses of ethnomedicinal plants among forest-dependent communities of Northern Bengal, India. Journal of Ethnobiology and Ethnomedicine 14:8.

Ramalingum N, Mahomoodally MF. The therapeutic potential of medicinal foods. 2014. Advances in Pharmacological Sciences 2014; 2014:354264. doi:10.1155/2014/354264

Sobirov RR, Kholmatov KhKh, Sobirov RS. 1993. Beruniy Shifobakhsh Giyohlar Khakida. Urganch. 40. (in Uzbek).

Sokolov SY, Zamotaev IP. 1984. Handbook of Medicinal Plants, Medicine Publishing House, 462. (in Russian).

Souza EN, Williamson EM, Hawkins JA. 2018. Which plants used in ethnomedicine are characterized? Phylogenetic patterns in traditional use related to research effort. Frontiers in plant science 9:834.

Taizhanov K, Kholmatov KhKh, Zhabborov A. 2003. Ar-Roziy foydalangan dorivor usimliklarning monavii tizimi. Kimyo va farmatsiya 2:10-14. (in Uzbek).

Taizhanov K, Taizhanova M. 2009. Taxonomic analysis of medicinal plants used in the Middle Ages East. Uzbek Biological Journal 6:43-47. (in Uzbek).

Tietel Z, Masaphy S. 2018. True morels (Morchella)-nutritional and phytochemical composition, health benefits and flavor: A review. Critical reviews in Food Science and Nutrition 58(11):1888-1901. doi: 10.1080/10408398.2017.1285269.

Tkachenko KG, Lebedeva TP. 2018. Ethnobotany in the modern world. Overview. Proceedings of Voronezh State University. Series: Chemistry. Biology. Pharmacy 2:172-184

Toyzhanov K, Kholmatov KhKh. 1998. Yusufiy Kullagan Dorivor Usimliklar. Kimyova Pharmacy. 2:22-25. (in Uzbek).

Vazirian M, Faridfar S, Eftekhari M. 2016. "Gharikon"/"Agharikon" a Valuable Medicinal Mushroom in Iranian Traditional Medicine. Iranian Journal of Medical Sciences 41(3):34.

Wang XW, Jiang JH, Liu SL, Gafforov Y, and Zhou LW. 2021. Species diversification of the coniferous pathogenic fungal genus Coniferiporia (Hymenochaetales, Basidiomycota) in association with its biogeography and host plants. Phytopathology. doi: 10.1094/PHYTO-05-21-0181-R

Wang, XW, Jiang J, Liu SL, Gafforov Y, Zhou LW. 2021. Species diversification of the coniferous pathogenic fungal genus Coniferiporia (Hymenochaetales, Basidiomycota) in association with its biogeography and host plants. Phytopathology. https://doi.org/10.1094/phyto-05-21-0181-R

Wasser SP, Coates P, Blackman M, Cragg G, Levine M, Moss J, White J. 2005. Encyclopedia of Dietary Supplements. New York: Marcel Dekker; Reishi or Lingzhi (Ganoderma lucidum). 680-690.

Wernig F, Buegger F, Pritsch K, Splivalloa R. 2018. Composition and authentication of commercial and home-made white truffle-flavored oils. Food Control 87:9-16. doi: 10.1016/j.foodcont.2017.11.045

Wolf EV, Maleeva OF. 1969. World resources of useful plants. Nauka Publishing House. 563. (in Russian).

Worldometers.info. 2021. https://www.worldometers.info/world-population/central-asia-population/ (Accessed 03/12/2021) 
Yatsiuk I, Saar I, Kalamees K, Sulaymonov S, Gafforov Y, O'donnell K. 2016. Epitypification of Morchella steppicola (Morchellaceae, Pezizales), a morphologically, phylogenetically and biogeographically distinct member of the Esculenta Clade from central Eurasia. Phytotaxa 284(1):31-40. doi: 10.11646/phytotaxa.284.1.3.

Živković J, Ilić M, Šavikin K, Zdunić G, Ilić A, Stojković D. 2020. Traditional Use of Medicinal Plants in South-Eastern Serbia (Pčinja District): Ethnopharmacological Investigation on the Current Status and Comparison with Half a Century Old Data. Frontiers in Pharmacology 11:1020. 